\title{
Die Rollen der Inskriptionen als nützliche Sichtweise im Mathematikunterricht
}

\author{
Martin Brunner
}

Eingegangen: 11. Oktober 2016 / Angenommen: 5. Januar 2017 / Online publiziert: 10. Februar 2017 (C) Der/die Autor(en) 2017. Dieser Artikel ist eine Open-Access-Publikation.

Zusammenfassung Im vorliegenden Aufsatz werden so genannte mathematische Darstellungen als Zeichenspiele im Sinne von Wittgenstein betrachtet. In diesen Zeichenspielen entsteht Bedeutung als Resultat der Verwendung von Inskriptionen nach bestimmten Regeln. Diese Verwendungsregeln werden zu Rollen der involvierten Inskriptionen zusammengefasst. Die angeführte Sichtweise bietet eine Fülle von Erklärungsmöglichkeiten für die Funktionsweise und das Zusammenwirken der so genannten mathematischen Darstellungen. Im Zuge der nachfolgenden Überlegungen werden Vorteile dieser Sichtweise durch Verweis auf theoretische Ansätze erläutert und anhand von Beispielen für die Praxis des Mathematikunterrichts nutzbar gemacht.

Schlüsselwörter Zeichenspiel · Inskription · Rolle $\cdot$ Regel $\cdot$ Bedeutung

Mathematics Education Subject Classification C $30 \cdot$ C $50 \cdot$ D $20 \cdot$ Q 30

\section{The role of inscriptions as a useful perspective in mathematics education}

Abstract In this paper so-called mathematical representations are considered as sign-games in the meaning of Wittgenstein. In these sign-games, meaning results from the use of inscriptions due to given rules. These usage rules are combined into roles of the involved inscriptions. This view offers a wealth of possible explanations for the function and the interaction of so called mathematical representations. In the course of the following considerations, advantages of this view are explained

\footnotetext{
M. Brunner

Universität Salzburg, Kapitelgasse 4-6, 5020 Salzburg, Österreich

M. Brunner $(\bowtie)$

Bundesrealgymnasium Lienz, Maximilianstr. 11, 9900 Lienz, Österreich

E-Mail: brunner.martin1@gmx.at
} 
theoretically and made available for the practice of teaching mathematics by means of given examples.

Keywords Sign-game $\cdot$ Inscription $\cdot$ Role $\cdot$ Rule $\cdot$ Meaning

\section{Einleitung}

Im vorliegenden Aufsatz wird eine semiotische Perspektive auf Mathematik eingenommen. Mathematische Darstellungen werden in Analogie zum Wittgenstein'schen Sprachspiel als Zeichenspiele betrachtet. Bedeutung entsteht in diesen Zeichenspielen durch die Verwendung von phonetischen und nonphonetischen Inskriptionen nach Regeln. Im Zentrum der Betrachtungen stehen dabei die Rollen der Inskriptionen. Sie sind durch die geltenden Verwendungsregeln im jeweiligen Zeichenspiel bestimmt. Beispielsweise ist etwa die Rolle einer geraden Linie als „Höhe“ im Zeichenspiel „Dreieck“ durch Verwendungsregeln wie „ohne Breite“ oder ,normal auf eine Seite in die gegenüberliegende Ecke“ bestimmt. Die jeweiligen Zeichenspiele und die Rollen der Inskriptionen in diesen Zeichenspielen bedingen einander. Gleiche Inskriptionen können zwar in verschiedenen Zeichenspielen unterschiedliche Rollen übernehmen, mit der Festlegung des Zeichenspiels sind aber die Rollen der Inskriptionen fixiert.

Der Begriff „Rolle“ wird im semiotischen und philosophischen Diskurs mit Bezügen zur Mathematik bzw. Mathematikdidaktik bereits in früheren Arbeiten verwendet. Als Urheber dieser Betrachtungsweise kann Wittgenstein gesehen werden. Wittgenstein (1984b, S. 67, vgl. Abschn. 2) spricht etwa von der Bedeutung des Wortes als „Rolle des Wortes“ im Sprachspiel und befasst sich explizit mit der Frage, in welcher Weise die Mathematik mit den Tintenstrichen auf dem Papier zu tun hat (vgl. Abschn. 2). Rotman (2000) postuliert drei Aspekte der Identität des Mathematikers, die er in den drei Rollen „Person“, „Subject“ und „Agent“ mit aufsteigender Abstraktheit in mathematischen Texten etabliert sieht. Brunner (2011, S. 11) beschäftigt sich mit der Rolle als „Darstellungsmuster“ und den verschiedenen Rollen, die etwa ein Vektor übernehmen kann. Dörfler (2013, S. 244) spricht von den Rollen von Zeichen und Wörtern in mannigfaltigen Sprachspielen der Mathematik. Die Rolle von Inskriptionen wird aber in den mir bekannten Arbeiten nie explizit untersucht und als didaktisches Hilfsmittel des konkreten Mathematikunterrichts betrachtet. Dies ist nun aber das Ziel des vorliegenden Aufsatzes. Nach der Präzisierung der Begriffe „Zeichenspiel“ und „Rolle von Inskriptionen“ geht es um eine konstruktive Deutung der Funktionsweise von so genannten mathematischen Darstellungen und deren Zusammenwirken. Verschiedene Formen der Bedeutungsfestlegung und Bedeutungsveränderung werden als Rollenfestlegungen, multiple Rollenverwendungen, Rollenübersetzungen, Rollennachweise usw. gedeutet. Das epistemologische Potential des Rollenwechsels wird ebenfalls anhand von Beispielen angedeutet. Die vorgeschlagene Sichtweise bietet viele Möglichkeiten, Analogien zur Erfahrungswelt der Lernenden im Mathematikunterricht gewinnbringend zu nutzen. Beispielsweise sind es Lernende gewohnt, in den verschiedenen „Zeichenspielen des Alltags“ Rollen wie jene als Klassensprecherin, Tochter, Schwester usw. zu spielen. Die Beleuchtung der 
mathematischen Darstellungen und des mathematischen Tuns mithilfe der Sichtweisen „Rolle“ und „Zeichenspiel“" kann vor allem für Lehrende aufschlussreich und hilfreich sein.

\section{Mathematik als Zeichenspiel}

Der Begriff des Zeichenspiels geht auf Wittgenstein zurück (vgl. etwa Wittgenstein 1984b, S. 257). Er ist an sein Sprachspiel angelehnt. Nach dem Wittgenstein'schen Sprachspiel haben Wörter und Sätze keine Bedeutung an sich. Die Bedeutung eines Wortes ist durch seine Rolle im Sprachspiel bestimmt. Mit Rolle ist die Art und Weise gemeint, nach welcher im Sprachspiel mit dem Wort kalkuliert wird. Die dabei geltenden Verwendungsregeln sind durch das Sprachspiel bestimmt. Wittgenstein (1984b, S. 67) schreibt:

Ich sagte, die Bedeutung eines Wortes sei die Rolle, die es im Kalkül der Sprache spiele. (Ich verglich es mit einem Stein im Schachspiel.) Und denken wir nun daran, wie mit einem Wort, sagen wir z. B. „,rot“ kalkuliert wird. Es wird angegeben, an welchem Ort sich die Farbe befindet, welche Form, welche Größe der Fleck oder der Körper hat, der die Farbe trägt, ob sie rein oder mit anderen vermischt, dunkler oder heller ist, gleich bleibt oder wechselt, etc. etc. Es werden Schlüsse aus den Sätzen gezogen, sie werden in Abbildungen, in Handlungen übersetzt, es wird gezeichnet, gemessen und gerechnet.

Nach Meyer (2010, S. 59) definiert Wittgenstein in diesem Sinne nicht, was er als Sprachspiel versteht. Er verleiht dem Wort Bedeutung, indem er Beispiele für den Gebrauch des Wortes anführt und den Umgang mit ihm beschreibt. Wittgenstein befasst sich auch explizit mit der Frage, in welcher Weise die Mathematik mit den Tintenstrichen auf dem Papier zu tun hat. Er erläutert seine Sicht mithilfe eines Vergleichs mit den Holzfiguren des Schachspiels (zitiert nach Epple 1994, S. 127):

Man hat mich in Cambridge gefragt, ob ich denn glaube, daß es die Mathematik mit den Tintenstrichen auf dem Papier zu tun habe. Darauf antworte ich: In genau demselben Sinn, wie es das Schachspiel mit den Holzfiguren zu tun hat. Das Schachspiel besteht nämlich nicht darin, daß ich Holzfiguren auf dem Holz herumschiebe. Wenn ich sage: ,Jetzt werde ich mir eine Königin anschaffen mit ganz furchtbaren Augen, die wird alles aus dem Feld schaffen", so werden Sie lachen. Es ist egal, wie ein Bauer aussieht. Es ist vielmehr so, daß die Gesamtzahl der Spielregeln den logischen Ort des Bauern ergibt.

Nach Epple (1994, S. 127) geht es hier um die Idee der Spielbedeutung. Diese Idee steht nach Wittgenstein im Widerspruch zu Freges bedeutungstheoretischer Sicht (zitiert nach Epple 1994, S. 127):

Für Frege stand die Alternative so: Entweder wir haben es mit Tintenstrichen auf dem Papier zu tun, oder diese Tintenstriche sind Zeichen von etwas, und das, was sie vertreten, ist ihre Bedeutung. Daß diese Alternative nicht richtig ist, zeigt gerade das Schachspiel: Hier haben wir es nicht mit den Holzfiguren 
zu tun, und dennoch vertreten die Figuren nichts, sie haben in Freges Sinn keine Bedeutung. Es gibt eben noch etwas drittes, die Zeichen können verwendet werden wie im Spiel.

Wittgenstein (1978, S. 171) ist aber nicht der Meinung, dass Mathematik ein Spiel ist:

Es ist sehr oft behauptet worden, die Mathematik sei ein Spiel, dem Schach vergleichbar. In einem Sinne ist diese Behauptung offensichtlich falsch: die Mathematik ist kein Spiel in der gewöhnlichen Bedeutung dieses Wortes. In einem anderen Sinn ist sie offensichtlich wahr: es gibt eine gewisse Ähnlichkeit. Nun sollte man aber nicht Partei ergreifen, sondern vielmehr eine Untersuchung anstellen. Manchmal ist es eben nützlich, die Mathematik mit einem Spiel zu vergleichen, und manchmal ist es irreführend.

Wittgenstein (2003, S. 56) sieht aber „Familienähnlichkeiten“, speziell im Zusammenhang mit dem Regelcharakter und der Bedeutungskonstitution. Im Sinne von Wittgenstein kann man daher mathematische Darstellungen als Zeichenspiele betrachten. In diesen Zeichenspielen werden Inskriptionen nach Regeln verwendet. Auch Teile von Darstellungen können wieder als eigene Zeichenspiele betrachtet werden. Die Verwendungsmöglichkeiten der Inskriptionen ändern sich in diesen „Teildarstellungen“ entsprechend. Beispielsweise können in einem Viereck zwei Seiten und eine Diagonale als Dreieck und damit etwa nach den Regeln des Cosinussatzes verwendet werden.

\section{Die Rollen der Inskriptionen}

Nach der im vorliegenden Aufsatz eingenommenen Sichtweise sind in den mathematischen Zeichenspielen die jeweiligen Rollen für die Inskriptionen bedeutungsbestimmend. Was kann nun über die Inskriptionen und ihre Rollen gesagt werden?

Im Zusammenhang mit mathematischen Inskriptionen gelten zunächst einmal Schreibregeln. Nicht jede beliebige Linie kann etwa als „gerade Linie“ verwendet werden. Es werden „Schreibtypen“ verwendet. Beispiele für einen Schreibtyp des Alphabets: a, a, a, a, a, a usw. Die für die die Zuordnung der infrage kommenden Elemente zu Schreibtypen (Klassen) erforderliche Herstellung von Beziehungen kann etwa mithilfe von Prototypentheorien beschrieben werden (vgl. etwa E. Rosch 1975 oder Lakoff 1987). Ein Prototyp ist dabei ein beispielhaftes Exemplar seiner Klasse. Ein Element wird nach derartigen Theorien als Mitglied einer Klasse gespeichert, wenn es dem Prototyp dieser Klasse ähnlicher als dem Prototyp einer anderen Klasse ist. Für Krämer (2009, S. 101) geht es bei der Identifikation von empirisch vorkommenden Inskriptionen als Verkörperung eines generellen Typus um eine Wiedererkennungsleistung, die auf der Vernachlässigung von Aspekten der sinnlichen Erscheinung beruht.

Inskriptionen haben generell keine feste Bedeutung. Beispiel: Eine Wellenlinie kann ein Zeichen für Wasser, für die Trennung von oben-unten, für eine Bewegung von links nach rechts, für eine Geste, für die Sinus- oder Cosinusfunktion, für 
Abb. 1 Der angeführte Strich kann auf unterschiedlichste Art und Weise Bedeutung erhalten

Periodizität usw. sein. Ein Zeichen zu sein kann nach dem Sprachgebrauch des vorliegenden Artikels als „Bedeutung erhalten durch“ interpretiert werden. Eine Wellenlinie kann im Wittgenstein'schen Sinn etwa dadurch eine spezielle Bedeutung erhalten, dass sie als Sinusfunktion verwendet wird.

Auch in der Mathematik haben Inskriptionen keine feste Bedeutung. Eine schreibregelkonforme gerade Linie (Abb. 1) hat etwa als „Strecke“ eine andere Bedeutung als als „Strahl“ oder „Gerade“. In allen drei Fällen wird sie als ,gerade“, ,ohne Breite“ und „ohne Höhe“ verwendet. Als Strecke wird sie als „Länge“ oder als ,kürzeste Verbindung zweier Punkte“ verwendet. Als Strahl wird sie als ,,von einem Anfangspunkt ausgehend“, „ohne Ende“ und als „orientiert“, als Gerade wiederum „ohne Anfangs- und Endpunkt“ und als ,unendlich lang“ verwendet. Für „Wissende“ werden durch einen Begriffsnamen wie „Strahl“ Verwendungen von Inskriptionen wie die angeführten zu Rollen zusammengefasst. In diesem Sinne sind die Rollen von Inskriptionen durch die Summe der geltenden Verwendungen und die dabei geltenden Regeln des jeweiligen Zeichenspiels bestimmt.

Gleiche Inskriptionen können in unterschiedlichen Zeichenspielen unterschiedliche Rollen übernehmen. Beispiele: Die Rolle der Inskription „2413“ ist in Zeichenspielen wie etwa dem ,Fünfer-“ oder ,Zehnersystem“ jeweils eine andere. Genauso wird die Inskription $\bigcirc$ in Zeichenspielen wie „Kreis“, ,runde Scheibe“ (das Innere beinhaltend), ,rundes Loch“ (das Innere nicht beinhaltend), „Kegelschnitt“, ,Jordankurve" usw. in jeweils anderen Rollen verwendet. Sogar in gleichen Zeichenspielen können gleiche schreibregelkonforme Inskriptionen nach verschiedenen Regeln und damit nach verschiedenen Rollen gebraucht werden. Beispiel: Eine gerade Linie kann im Zeichenspiel „Dreieck“ etwa Rollen wie „Seite“, „Höhe“, „Schwerelinie“, „Seitensymmetrale“, oder „Winkelsymmetrale“ übernehmen. Zur Unterscheidung der verschiedenen Rollen sind daher nicht nur die Namen der jeweiligen Zeichenspiele, sondern auch die Namen der jeweiligen Rollen wichtig. Die Rollen der Inskriptionen und das jeweilige Zeichenspiel bedingen einander. Welche Inskriptionen in den jeweiligen Zeichenspielen nach bestimmten Rollen verwendet werden, muss aus Vertrautheit mit den jeweiligen Zeichenspielen erwachsen. Nicht jede Inskription übernimmt nach den Regeln des Zeichenspiels eine eigene Rolle. Beispiel: Der Buchstabe , $\mathrm{i}^{\text {“ }}$ übernimmt in $f(\mathrm{i})=\mathrm{ai}^{2}+\mathrm{bi}+\mathrm{c}$ eine eigene Rolle, in ,sinx“ wird er aber traditionsgemäß nicht in einer eigenen mathematisch relevanten Rolle verwendet. Er bezieht seine Bedeutung nur durch seine Funktion als Teil des Namens ,sin“.

Die Verwendung von Inskriptionen nach bestimmten Rollen schließt andere Rollen aus (vgl. Brunner 2015b). Das hat Konsequenzen. Verwendet man etwa eine Linie als ,gerade“, so kann sie nicht gleichzeitig etwa als ,rund“, verwendet man ein Zahlzeichen als ,gerade“, so kann es nicht gleichzeitig als ,ungerade“ verwendet werden. Verwendet man Striche im Zusammenhang mit einem Rechteck ohne „Breite“, so muss man sich etwa bei der Berechnung der Fläche um die Strichbreite keine Gedanken machen. Beim Wechsel des Zeichenspiels müssen Rollen 
Abb. 2 Die angeführten Inskriptionen spielen im $\mathrm{IR}^{3}$ andere Rollen als im $\mathrm{IR}^{2}$

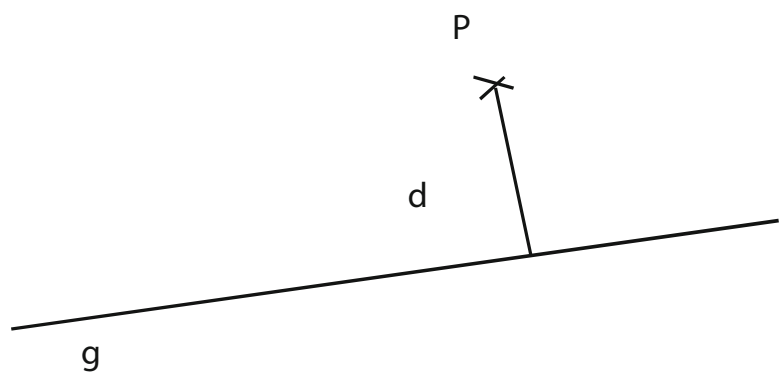

von Inskriptionen übersetzt werden. Es müssen die jeweiligen Verwendungsregeln angepasst werden. Änderungen bei den Rollen von Inskriptionen bedingen Änderungen, Fortschreibungen, Erweiterungen bzw. Verengungen bei den Rollen anderer Inskriptionen und in der Folge andere Lösungsmöglichkeiten im Zusammenhang mit gestellten Problemen. Beispiel: Verwendet man die folgenden Inskriptionen nach den Rollen im Zeichenspiel $\mathrm{IR}^{3}$ anstelle des $\mathrm{IR}^{2}$, so müssen etwa zur Berechnung des Normalabstandes d andere Verfahren gewählt werden (siehe Abb. 2).

Die Rolle der geraden Linie als Normalvektor im $\mathrm{IR}^{3}$ ist eine andere als im IR 2 .

Durch die im vorliegenden Aufsatz bezogene Sichtweise von Rolle und Zeichenspiel kommt dem Regelbegriff zentrale Bedeutung zu. Er ist im vorliegenden Aufsatz an jenem von Wittgenstein orientiert. Hoffmann A. (2007, S. 1) schreibt über den Wittgenstein'schen Regelbegriff:

Regeln [...] werden nicht durch die logische Summe ihrer Beispiele explizit definiert. Die Regel wird durch eine nicht weiter hintergehbare Ähnlichkeit gegeben, die unter den zu ihrer Definition angegebenen Beispielen besteht. Diese Ähnlichkeit wird durch jedes neu hinzukommende Beispiel fortgeschrieben, verengt, verändert oder auch erweitert. [...] Das Erlernen von Regeln beinhaltet daher immer zwei Punkte: Einerseits die Erkennung ihrer Anwendungskriterien in konkreten Situationen, d.h. die Subsumption einer Erfahrung unter der jeweiligen Regel. Andererseits die spezifische Fortschreibung, die Veränderung, die Verengung oder Erweiterung der Regel aufgrund jeder neuen Erfahrung.

Korrekte Regelanwendung ist speziell im Zusammenhang mit Mathematik nicht einfach. Im Sinne des obigen Zitats muss man beispielsweise wissen, welche Regeln im Zusammenhang mit welchen Rollen von Inskriptionen überhaupt Gültigkeit besitzen. Zusätzlich muss man erkennen, welche Anwendungskriterien in einer konkreten Situation die Verwendung einer bestimmten Regel rechtfertigen. Auf die Notwendigkeit der ständigen Regeldifferenzierung in der Form von Regelfortschreibungen, Regelverengungen, Regelerweiterungen oder Regelveränderungen wurde bereits beim obigen Beispiel verwiesen. In diesem Sinne erfordert die Fähigkeit korrekter Regelauslegung in der Mathematik die Subsumption vieler verschiedener Spezialfälle. Regelanwendung ist also selbst nicht durch eine allgemein gültige Regel beschreibbar. 


\section{Rolle statt Repräsentation}

Nach Dörfler (2014, S. 72) beschreiben mathematische Zeichen nach der Wittgenstein'schen Sichtweise keinen vorgegebenen Gegenstand. Er schreibt (2014, S. 72):

Die Zeichen haben in der Mathematik auch keinen vorgegebenen Gegenstand, für den sie stehen oder den sie bezeichnen. Mathematik hat in diesem Sinne keinen außerhalb von ihr gelegenen Gegenstand, sie ist ein System von Regeln (Normen, wie Wittgenstein sagt).

Als Beleg führt Dörfler etwa das folgende Zitat von Wittgenstein (1984a, S. 468) an:

In der Mathematik ist alles Algorithmus, nichts Bedeutung; auch dort, wo es scheint, weil wir mit Worten über die mathematischen Dinge zu sprechen scheinen. Vielmehr bilden wir dann eben mit diesen Worten einen Algorithmus.

Dies unterscheidet die angeführte Sichtweise von der üblichen platonistischen. Bei der platonistischen Sicht werden Inskriptionen als Darstellungen von idealen abstrakten Inskriptionen und Zeichenspiele als Darstellungen von nicht wahrnehmbaren abstrakten Objekten betrachtet. Die platonistische Sichtweise ist referentiell. Ein gezeichneter Strich verweist etwa als Darstellung einer „Geraden“ auf eine unendlich lange, ideal gerade Linie ohne Breite. Nach der platonistischen Sichtweise gibt es daher die Tendenz, zwischen essentiellen Inskriptionen und solchen, die nur „Beiwerk“ sind, zu unterscheiden. Es gibt Darstellungen, in welchen bestimmten schreibregelkonformen Inskriptionen „Objektcharakter“ zugebilligt wird, und solche, in welchen den gleichen Inskriptionen dieser Charakter abgesprochen wird. Beispielsweise stellt die gerade Linie als Strecke, Strahl, Gerade, Höhe, Schwerelinie, Winkelhalbierende, Kante, Diagonale usw. abstrakte Objekte dar. Anders verhält es sich mit der gleichen Inskription als Betragsstrich, Normstrich, Bruchstrich, Abstandsstrich usw. Ich unterstelle, dass diese Betrachtungsweise auch Auswirkungen auf die Art der Behandlung der Inskriptionen als Teile der verschiedenen Darstellungen im Unterricht hat. Bei der im vorliegenden Aufsatz vorgeschlagenen Sichtweise sind im Gegensatz zur platonistischen Sicht alle Arten von Inskriptionen für die jeweiligen Zeichenspiele in gewisser Weise gleich wichtig. Auch die Rollen von Betragsstrichen, Normstrichen, Bruchstrichen, Abstandsstrichen usw. sind für den regelkonformen Umgang mit Zeichenspielen wie $|x+2|>-2$ essentiell. Es rücken damit die involvierten Inskriptionen und alle Arten von Verwendungsregeln in den Vordergrund.

Die platonistische Sichtweise bringt speziell im Zusammenhang mit der Didaktik der Mathematik Probleme mit sich. Nach Dörfler (2006) ist sie eine der Ursachen für die empirisch belegbare Angst vor Mathematik. Mühlhölzer (2012, S. 10) betrachtet die Objektsprache, die der Interpretation bedarf, als tot. Sie wird selbst nicht verwendet. Nur die Zeichen, die wir diesen Objekten zuschreiben, werden verwendet. Er schreibt:

The object language that is interpreted - is not a language considered as used. It is a purely mathematical object, and mathematical objects are not used: 
only the signs with which we refer to these objects are used.[...] in case of an interpretation, the object language that is interpreted is dead like any other mathematical object ...

Mühlhölzer (1999) kritisiert darüber hinaus den in der Mathematik weitverbreiteten Platonismus generell und plädiert im Wittgenstein'schen Sinn für eine Mathematik ohne ,,platonistische Metaphysik“. Es können darüber hinaus leicht Schwierigkeiten thematisiert werden, die aus der angesprochenen Metaphysik resultieren. Beispielsweise suggeriert der mathematische Sprachgebrauch die implizite Wirksamkeit der abstrakten Objekte. Beispiel: Exponentialfunktionen $\left(y=a^{x}\right)$ wachsen für $x \rightarrow \infty$ stärker (schneller) gegen unendlich als Potenzfunktionen $\left(y=x^{n}\right)$. Diese Aussage suggeriert, dass der angesprochene Sachverhalt wegen der entsprechenden Objektzugehörigkeit eben als Exponentialfunktion bzw. Potenzfunktion gilt. Nach der im vorliegenden Aufsatz vertretenen Sichtweise resultiert der angesprochene Sachverhalt aber aus den entsprechenden Inskriptionsverwendungen und damit aus den jeweiligen Rollen der Inskriptionen.

Nach der platonistischen Sicht wird auch suggeriert, dass es im Zusammenhang mit den abstrakten Objekten trotz ständigen Bedeutungsänderungen so etwas wie einen gleichbleibenden Bedeutungskern geben muss. Man spricht ja in unterschiedlichsten Kontexten vom „Punkt“, von der „Geraden“, vom „Dreieck“, von der „Zahl 5“ usw. Bereits das Dreieck ist aber ein Gegenbeispiel dieser Theorie eines gleichbleibenden Bedeutungskerns. Vergleicht man alle verschiedenen Dreiecke miteinander, so gibt es letztlich außer dem gemeinsamen Namen keine einzige Eigenschaft, die allen Dreiecken von der euklidischen Geometrie bis hin zur Graphentheorie gemeinsam ist. Der angesprochene Bedeutungswandel erfordert zudem die ständige Restrukturierung der Bedeutung der Begriffe im Verlauf des mathematischen Lernprozesses (vgl. Brunner 2011). Wird dieser ständige Bedeutungswandel nicht ausreichend bearbeitet, so führt dies zu einer Verunsicherung der Lernenden. Es bleibt immer ein „unverstandener“ Rest. Die im vorliegenden Aufsatz beschriebene nicht referentielle Sichtweise von Rolle und Zeichenspiel bietet auch im Hinblick auf diesen Bedeutungswandel didaktische Vorteile. Begriffe beziehen ihre Bedeutung nach der Sichtweise des vorliegenden Artikels aus den subsumierten mathematischen Zeichenspielen (siehe nächster Absatz). Es kann damit alles offengelegt werden: Inskriptionen, Inskriptionsverwendungen und Regeln. Es bleibt kein unbestimmter Rest. Die Inskriptionsebene ist selbst die beobachtbare Ebene mathematischen Tuns. Bedürftig (2016) thematisiert die Präzision, welche der sorgfältige didaktische Umgang mit Begriffen erfordert. Er zeigt detailliert die Bedeutungsänderungen, die der Begriffsname „Punkt“ und die Inskription „Punkt“ von der euklidischen Definition bis hin zum Objekt „Punkt“, in dessen Umgebung sich unendlich viele reelle und hyperreelle Zahlen befinden, erfährt. Im Sinne von Bedürftig (2016, S. 15) sind wir es, die an einer bestimmten Stelle „Stopp“ sagen. An einer bestimmten Stelle der Lehrstoffentwicklung im Unterricht verwenden wir die Inskription „Punkt“ und die Sprechweise „Punkt“ nach der Diktion des vorliegenden Artikels in klar definierten Rollen, an einer anderen Stelle eben zumindest partiell anders.

Aus dem Wittgenstein'schen Bedeutungskonzept resultiert eine weitere didaktische Konsequenz. Die Bedeutung der mathematischen Sprechweisen kann nur aus 
der Vertrautheit mit den entsprechenden Zeichenspielen und den Rollen der Inskriptionen entstehen. Die Korrektheit einer sprachlichen Formulierung kann ohne Vertrautheit mit den entsprechenden mathematischen Zeichenspielen und den Rollen der Inskriptionen nicht entschieden werden. Aussagen wie „Die Zahl 5 schneidet das Dreieck“ oder „Zwei Geraden der Ebene sind parallel und stehen aufeinander normal" sind, wie man sieht, sprachlich ohne jede Widerständigkeit formulierbar, skriptural sind sie aber unter Verwendung der Regeln der Mathematik nicht formulierbar. Es ist also nicht möglich, ,vorab“ mithilfe mathematischer Sprechweisen Bedeutung zu vermitteln. Man muss im Mathematikunterricht einerseits lernen, wie man mit den Inskriptionen des jeweiligen Zeichenspiels umgeht, und andererseits, wie man über dieses Tun spricht. Nur auf Basis der angesprochenen Vertrautheit mit den jeweiligen Zeichenspielen kann der mathematische Sprachgebrauch zu einem Sprachspiel mit epistemologischem Potential werden. Hier ist etwas von Bedeutung, das Wittgenstein (1984b, S. 84) ,die Härte des logischen Muß“ genannt hat. Korrektes mathematisches Tun, bestimmt durch das jeweilige Zeichenspiel und die darin geltenden Rollen der Inskriptionen, führt unausweichlich zu bestimmten Erfahrungen (vgl. etwa Brunner 2015a). Alle Mathematiker/innen, welche die involvierten Inskriptionen korrekt verwenden, werden gleiche Ergebnisse erhalten. Es muss die Unausweichlichkeit des korrekten mathematischen Tuns erfahrbar sein, um diese Unausweichlichkeit auf das mathematische Sprachspiel übertragen zu können.

Mithilfe der Rolle der Inskriptionen kann auch gut vermittelt werden, dass geänderte Verwendungsregeln zu geänderten Sprechweisen führen. Dies kann möglicherweise bereits durch einfache Beispiele wie das folgende den Lernenden zumindest auf einer intuitiven Ebene vermittelt werden: Das Zahlzeichen ,5“ ist etwa als natürliche Zahl durch seine Rolle bestimmt. Würde man die Zahlzeichen etwa so anordnen: $0,2,1,4,3,6,5$, ..., so wäre ,5“ eine gerade Zahl und es würden sich vollkommen andere „Darstellungsmöglichkeiten“ der Zahl ,5“, wie etwa „,4 + 4 = 5“, ergeben. Man würde aber weiterhin von der natürlichen Zahl ,5“ sprechen. Diese Sprechweise hätte aber eine andere Bedeutung. Erklärungsweisen wie die angeführte sind für Lernende möglicherweise leichter verstehbar als jene, dass gleiche Inskriptionen potentiell verschiedenste abstrakte Objekte repräsentieren können.

Mithilfe der im vorliegenden Aufsatz propagierten Sichtweise von Zeichenspiel und Rolle können auch weitere Eigenheiten des üblichen Sprachgebrauchs gut sichtbar gemacht werden. Hier einige Beispiele: Zunächst gibt es aufgrund der vorherrschenden Sichtweise von mathematischen Inskriptionen als Darstellungen abstrakter Objekte immer wieder verschiedene Begriffsnamen für das „Gleiche“. Beispiel: „Strecken“ heißen in ebenen Figuren „Seiten“ oder als Bestandteile bestimmter Winkel „Schenkel“. Verwendungsregeln (Rollenbestandteile) werden zudem als Eigenschaften abstrakter Objekte dargestellt. Beispiel: Eine Dreieckshöhe steht normal auf eine Seite. Auch beobachtbare Auswirkungen (abgeleitete Regeln) von Verwendungsregeln werden als Eigenschaften von abstrakten Objekten dargestellt. Beispiel: „5“ ist eine ungerade Zahl (wie oben ausgeführt ist diese „Eigenschaft“ lediglich das Resultat einer bestimmten Anordnung der Zahlzeichen).

Obwohl die Zeichenspiele der Mathematik im vorliegenden Aufsatz nicht als Darstellungen abstrakter Objekte betrachtet werden, wird im Folgenden die übliche Sprechweise „Darstellung“ verwendet. 


\section{Multiple Rollenverwendung und die Vernetztheit der Zeichenspiele}

In Arbeiten wie Brunner (2015b) wurden bereits Aspekte des Zusammenwirkens der mathematischen Zeichenspiele und der Inskriptionsverwendungen beleuchtet. Nach der Diktion des vorliegenden Aufsatzes wurde dargelegt, dass durch die Kombination von Inskriptionen und deren multipler Verwendung nach unterschiedlichen Rollen die jeweiligen Zeichenspiele vielfältig verwendbar und multipel miteinander vernetzt sind. Es können auf diese Art und Weise sogar Phänomene rein konstruktiv erklärt werden, die gemeinhin als Beleg für die Sinnhaftigkeit einer platonistischen Sichtweise auf mathematische Darstellungen und für die Existenz abstrakter Objekte betrachtet werden. Ein solches Phänomen ist etwa die so genannte multiple Repräsentation von abstrakten Objekten. Gemeint ist damit, dass es in der Regel mehrere Zeichenspiele gibt, die mathematisch aufeinander bezogen sind und nach der platonistischen Sichtweise das gleiche abstrakte Objekt darstellen. Beispielsweise wird nach dieser Sichtweise das abstrakte Objekt „komplexe Zahl“ durch Zeichenspiele wie „a + bi“, Gauß'sche Zahlenebene, Polarkoordinaten, Matrizendarstellung und Riemann'sche Zahlenkugel repräsentiert. Dieses Phänomen kann aber rein konstruktiv erklärt werden. Im Folgenden wird das Zusammenwirken von „a + bi“ mit der Gauß'schen Zahlenebene mithilfe der Rollen von Inskriptionen konstruktiv erklärt (in Anlehnung an Brunner, S. 2013, S. 64). Bei der Gauß'schen Zahlenebene werden zwei schreibregelkonforme gerade Linien zunächst in den Rollen „Gerade“ (hier Achse genannt) und „rechter Winkel“ genommen. Durch die Deutung von „1“ und „,““ als Einheitslängen können die reellen Zahlen auf der 1. Achse und die imaginären Zahlen auf der 2. Achse veranschaulicht sowie die entsprechenden Punkte auf den Achsen in den Rollen von reellen Zahlen bzw. imaginären Zahlen verwendet werden. Durch diese Art der Inskriptionseinbettung können geometrische Gebilde algebraisch gedeutet werden. Die Verknüpfung „,+“ erhält erst jetzt eine Bedeutung. Das Strukturzeichen ,+" hat ja in „,a + bi“ keine mathematisch relevante Bedeutung. „a“ und „bi“ können algebraisch nicht so addiert werden, dass „,+" verschwindet. Das Pluszeichen spiegelt hier in erster Linie den Entstehungskontext in Form konkreter Gleichungslösung wider. Es steht für die Paarbildung. Es ist neben „i“ ein Erkennungsmerkmal einer komplexen Zahl. Durch die beschriebenen konstruktiven Maßnahmen können nun „Stellen“ auf dem Zeichenblatt die Rolle von Punkten in der Gauß'schen Zahlenebene übernehmen. Das Zeichenspiel „Gauß'sche Zahlenebene" kann durch die beschriebene Inskriptionskombination um mathematische Rollen wie Winkel, Richtung oder Vektoren erweitert werden. Die Ebene wird als Vektorraum mit der Basis $\{1, \mathrm{i}\}$ verwendbar. Durch die Art der Inskriptionskombination führen Operationen wie $(\mathrm{a}+\mathrm{bi})+(\mathrm{c}+\mathrm{di})$ bei algebraischer und bei geometrischer Ausführung zu scheinbar gleichen Ergebnissen. Durch diese Erweiterung können gleiche Objekte wie etwa „Punkte“ durch unterschiedliche relationale Zusammenhänge wie $(\mathrm{a} / \mathrm{b})$ und $(\mathrm{r} / \varphi)$ (Polarkoordinaten) bestimmt werden. Der bestehende Isomorphismus zwischen $(\mathrm{a} / \mathrm{b})$ und $(\mathrm{r} / \varphi)$ erlaubt die Zuordnung der beiden Darstellungen zum gleichen mathematischen Objekt ,komplexe Zahl“. Nach Dörfler (2015, S. 37) ist der angesprochene Isomorphismus der verschiedenen Zeichensysteme der komplexen Zahlen nur die algebraische Formulierung der beobachtbaren Tatsache, dass jedem Zeichen eines Systems eineindeutig ein Zeichen des anderen Systems 
entspricht. Die Transformation von $(\mathrm{a} / \mathrm{b}) \mathrm{zu}(\mathrm{r} / \varphi)$ geht mit einer anderen Sichtweise einher, die auch Auswirkungen hat. Beispielsweise ist nun' und $\varphi+2 \pi$ dieselbe Zahl $\mathrm{z} \in \mathcal{\complement}$ zugeordnet oder es besteht nun auch der bekannte Zusammenhang zwischen der Multiplikation komplexer Zahlen und der Addition der zugehörigen Winkel: ,, $\mathrm{z}_{1} \cdot \mathrm{z}_{2}=\mathrm{r}_{1} \cdot \mathrm{r}_{2}\left(\left(\cos \varphi_{1}+\varphi_{2}\right)+\mathrm{i} \cdot \sin \left(\varphi_{1}+\varphi_{2}\right)\right)$ “. Die Festlegung von Punkten durch Polarkoordinaten eröffnet wiederum neue Möglichkeiten. Beispielsweise kann ein weitreichender Zusammenhang zwischen trigonometrischen Funktionen und der Exponentialfunktion hergestellt werden (Potenzieren, Wurzelziehen usw.).

Mathematische Inskriptionen sind nach der Semiotik von Peirce „Diagramme“. Mit „Diagrammatik“ bezeichnet man jene Teildisziplin der Semiotik, die sich generell mit Diagrammen und der Erkenntnisentwicklung mithilfe von Diagrammen beschäftigt. Ich verweise in diesem Zusammenhang auf Arbeiten wie Nagl (1992); Hoffmann (2005); Dörfler (2006); bzw. Dörfler und Kadunz (2006); Stjernfelt (2007); Brunner (2009), (2011). Mithilfe der Sichtweise von Rolle und Zeichenspiel kann den Lernenden Essentielles der Diagrammatik nach Peirce, etwa in den Diagrammen wirksame Regeln, auf einer intuitiven Ebene vermittelt werden. Es gibt beispielsweise Regeln, die für die Geschlossenheit und die Abgrenzbarkeit eines Zeichenspiels sorgen. Drei schreibregelkonforme gerade Linien sind etwa nur dann als Dreieck verwendbar, wenn sie als geschlossener Streckenzug gebraucht werden können. Die gleiche Bedingung gilt, wenn man drei schreibregelkonforme gerade Linien eines Vierecks als Dreieck verwenden will (zwei Seiten und eine Diagonale). Durch die angeführte Regel ist das Dreieck vom Viereck abgrenzbar und für sich geschlossen. Im vorliegenden Aufsatz werden derartige Regeln als „systemische Regeln“ bezeichnet. Durch die Geschlossenheit der Zeichenspiele und die relationale Verwendung der Inskriptionen kann man Zeichenspiele als geschlossene relationale Systeme betrachten. In solchen Systemen kann man mithilfe von Experimenten Kenntnisse gewinnen. Beispiel: Im geschlossenen System „Dreieck“ werden je zwei Seiten nach dem Zeichenspiel „Winkel“" verwendet. „Winkel“ ist dabei eine Sprechweise für eine bestimmte relationale Verwendungsweise der involvierten Inskriptionen. Durch die Geschlossenheit des Dreiecks sind die drei Winkel voneinander abhängig. Änderungen bei einem Winkel führen zu Änderungen bei mindestens einem anderen Winkel. Es sind aber nicht nur die Winkel betroffen. Wegen der multiplen Verwendbarkeit der Inskriptionen nach Rollen ergeben sich Auswirkungen auch im Zusammenhang mit anderen Rollen und anderen Zeichenspielen: Verwendet man alle Winkel eines Dreiecks als gleich groß, so kann man bei entsprechender Vertrautheit mit den jeweiligen Zeichenspielen feststellen, dass alle Seiten gleich lang sind, die Berechnung der Fläche nach der Formel $\mathrm{A}=$ $\frac{a^{2}}{4} \cdot \sqrt{3}$ erfolgen kann, Umkreismittelpunkt, Schwerpunkt und Höhenschnittpunkt zusammenfallen usw..

Die Mittel des Kenntnisgewinns sind dabei Experiment und Beobachtung. Zeichnet man etwa wiederholt Umkreismittelpunkt, Schwerpunkt und Höhenschnittpunkt in gleichseitige Dreiecke ein, so wird man bei entsprechender Genauigkeit zur Beobachtung kommen, dass die drei Punkte zusammenfallen. Mithilfe geeigneter Transformationen können derartige Vermutungen dann bewiesen werden. Im Gegensatz zu empirischen Experimenten der Naturwissenschaften stimmen derartige Kenntnisse in der Mathematik immer. Es gibt in den Zeichenspielen der Mathematik keine 
Unbestimmtheiten. Führt man mit schreibregelkonformen Inskriptionen in bestimmten Rollen bestimmte Transformationen regelkonform durch, so führt dies unausweichlich zu bestimmten Ergebnissen. Diese Ergebnisse sind abgeleitete Regeln, die Vorhersagen erlauben und in der Folge das mathematische Handeln im Umgang mit entsprechenden mathematischen Zeichenspielen anleiten können. Wittgenstein formuliert das so (1984b, S. 359):

„Wenn du mit diesen Zahlen machst, was ich dir mit anderen Zahlen vorgemacht habe, wirst du ... erhalten“ - das heißt doch: „Das Resultat dieser Rechnung ist ..." - und das ist keine Vorhersage, sondern ein mathematischer Satz. Aber es ist dennoch auch eine Vorhersage! - Eine Vorhersage besonderer Art. Wie der, der am Ende findet, daß sich beim Addieren der Kolumne wirklich das und das ergibt, wirklich überrascht sein kann; z. B. ausrufen kann: ja, bei Gott es kommt wirklich heraus! Denke dir nur diesen Vorgang des Vorhersagens und der Bestätigung als ein besonderes Sprachspiel - ich meine: isoliert von dem Übrigen der Arithmetik und ihrer Anwendung.

Eine didaktische Herausforderung bleibt die Vermittlung der Relationalität mathematischer Zeichenspiele. Verwendet man Inskriptionen nur „figürlich“, so kann das zu Problemen beim Übergang von der empirischen Begriffsentwicklung führen (vgl. Dawydow 1977; Dörfler 1988; Peschek 1989). Beispiel: Verwendet man beim Zeichenspiel „Kreisgleichung“ anstelle der euklidischen Metrik die Maximumsmetrik, so wird beim entsprechenden geometrischen Zeichenspiel die „Kreisfigur“ zur „Quadratfigur“. Es ändert sich die schreibregelkonforme Inskription. Die Rolle bleibt gleich. Auch die „Quadratfigur“" steht für die Menge aller Punkte einer Ebene, die einen konstanten Abstand zu einem vorgegebenen Punkt dieser Ebene, dem Mittelpunkt, haben. „Figürlichkeit“ ist also keine mathematisch relevante Rolle von Inskriptionen.

Mathematische Zeichenspiele können auch als Regelsysteme betrachtet werden, die den Gebrauch der involvierten Inskriptionen bestimmen. Solche Regelsysteme können in der Mathematik auch losgelöst von entsprechenden „Verkörperungen“ angegeben werden. Betrachtet man als Beispiel die Axiome der natürlichen Zahlen nach G. Peano, so sieht man, dass die bekannten Axiome in diesem Sinne als „Rollenregeln“ und systemische Regeln interpretiert werden können. Man könnte die Regeln so zusammenfassen: Rolle 1: „0“ ist eine natürliche Zahl und „,0“ ist kein Nachfolger einer natürlichen Zahl. Rolle 2: Jede natürliche Zahl hat eine natürliche Zahl als Nachfolger. Systemische Regeln: Natürliche Zahlen mit gleichem Nachfolger sind gleich. Enthält X die ,0“ und mit jeder natürlichen Zahl $n$ auch deren Nachfolger $n^{\prime}$, so bilden die natürlichen Zahlen eine Teilmenge von X. Zeichenspiele, in welchen diese Regeln ,wirksam“ sind, können als natürliche Zahlen verwendet werden. Es sind unterschiedliche „Verkörperungen“ dieses Regelsystems denkbar. Beispiele:

I, II, III, IIII, IIIII, IIIIII, ...

$0,1,2,3,4,5,6,7,8,9,10,11,12, \ldots$ 
Zeichenspiele wie die angeführten erfüllen sowohl die Rollenregeln als auch die systemischen Regeln. Beispielsweise ist eine Konsequenz der Axiome jene, dass Zeichensysteme, die als natürliche Zahlen verwendet werden können, Regeln der beliebig fortsetzbaren Zeichengenerierung beinhalten müssen. Die obigen Beispiele erfüllen diese Forderung. Römische Zahlzeichen würden hingegen keine ungehinderte Zeichengenerierung ermöglichen. Obwohl beide obigen Zeichenspiele die Anforderungen des Axiomensystems erfüllen, sind sie nicht gleichwertig. Das obere Zeichensystem verwendet nur eine einzige schreibregelkonforme Inskription, das untere zehn verschiedene. Es ergeben sich daraus und aus den geltenden Regeln die bekannten Vorteile des unteren Systems: mehr Überblick, bessere Handhabbarkeit und bessere Ausbaubarkeit.

\section{Rollenfestlegungen und Rollenbeschreibungen}

Viele mathematische Verfahren der Bedeutungsfixierung wie etwa Definitionen können mithilfe der vorgeschlagenen Sichtweise als Rollenfestlegungen betrachtet werden.

Wie bei den Regeln eines Spiels darf es auch bei jenen der Mathematik keine Ungeklärtheiten geben. Wittgenstein (2003, S. 77) schreibt: „Es ist doch kein Spiel, wenn es eine Vagheit in den Regeln gibt.“ Dementsprechend müssen die jeweiligen Inskriptionsrollen der mathematischen Zeichenspiele genau festgelegt sein. Rollenfestlegungen erfolgen in der Mathematik häufig in eigenen Zeichenspielen. Beispiel: Das nachfolgend angeführte Zeichenspiel regelt beispielsweise die Verwendung der Betragsstriche:

$$
|x|:=\left\{\begin{array}{c}
x \text { für } x \geq 0 \\
-x \text { für } x<0
\end{array}\right.
$$

Wie bereits erwähnt, muss auch das Zusammenwirken von Inskriptionsrollen genau durch Regeln festgelegt sein. Derartige Regeln werden häufig einfach verbal formuliert. Beispielsweise wird das Zusammenwirken zweier schreibregelkonformer Linien, die in den Rollen Kreis und Tangente verwendet werden, durch die Grundregel „Kreis und Tangente haben nur einen Punkt gemeinsam“ bestimmt. Im Sinne von Hjelmslev (Mahr und Robering 2009, S. 300) handelt es sich hier um Präzisierungen und nicht um Exemplifizierungen von Verwendungsregeln oder gar um die Beschreibung einer „Realität“. Viele Rollenfestlegungen regeln die Ersetzung der involvierten Inskriptionen. Beispiel: $(-1)(-1)=1$. Derartige Grundregeln können nicht „,verstanden“ werden. Man kann eventuell im Zusammenhang mit den verschiedenen mathematischen Zeichenspielen ihre Zweckmäßigkeit begreifen, man kann sie ansonsten aber nur befolgen. Beim gegebenen Beispiel ist die Zweckmäßigkeit etwa durch die Erfüllung von Körperaxiomen gegeben. Neben der Festlegung ist auch die Abgrenzung von Rollen von Bedeutung. Skripturale Unterschiede weisen häufig auf Rollenunterschiede hin. Beispiel: Die Rollen von $\mathrm{x}$ und $\mathrm{y}$ sind in $\sqrt{x}+\mathrm{y}$ andere als in $\sqrt{x+y}$. Es lassen sich in diesem Zusammenhang auch Parallelen bzw. Unterschiede zwischen mathematischer Inskriptionsverwendung und Alltagssprache 
beobachten. Schweiger (2015, S. 135) untersucht solche Unterschiede etwa im Hinblick auf die Klammersetzung. Nach Schweiger sind in der Mathematik Klammern fast unvermeidbar, während sie in der Alltagssprache nicht oder kaum vorkommen. Beispiel (Schweiger 2015, S. 139): Das Kompositum „Handschuhfach“ ist etwa kein Schuhfach für Hände. Man setzt hier nicht wie in der Mathematik Klammern der Form „(Handschuh)fach“, so wie man eben in der Mathematik $(x+2) \cdot 3$ von $x+2 \cdot 3$ unterscheidet. Häufig beziehen sich Zeichenspiele der Mathematik gerade im Hinblick auf bestimmte Rollen aufeinander. Rollen von Inskriptionen in einem Zeichenspiel können durch ein anderes Zeichenspiel sichtbar gemacht werden. Beispiel: Im Zeichenspiel $\mathrm{a}^{\mathrm{x}}=\mathrm{b}$ haben $\mathrm{a}, \mathrm{x}$ und $\mathrm{b}$ verschiedene Rollen. Will man $\log _{\mathrm{a}} \mathrm{b}$ verstehen, so muss man zunächst die spezielle Rolle von $\mathrm{x}$ in $\mathrm{a}^{\mathrm{x}}=\mathrm{b}$ verstehen.

Unterschiedliche Beschreibungsmöglichkeiten gleicher Rollen sind in der Mathematik von großer Bedeutung. Hier zunächst Beispiele zu unterschiedlichen Rollenbeschreibungen innerhalb eines Zeichenspiels: Mithilfe von Ersetzungs- und Operationsregeln und dem Ausbau des Zeichenspiels mithilfe von Inskriptionskombinationen kann das Dezimalsystem auf die bekannte Art und Weise ausgebaut werden. Es gelten Inskriptionsersetzungen wie $5=6-1=-2+7=40: 8=4,7+0,2+$ $0,1=\frac{17}{3}-\frac{2}{3}=6, \dot{6}-1, \dot{6}=$ usw. Die Rolle ,5“ kann nun auf viele Arten beschrieben werden. Jede dieser Beschreibungen ist eine Kenntnis, die wiederum bei anderen Rollenbeschreibungen verwendet werden kann. Beispiel: $5+3=40$ : $8+3$. Rollenbeschreibungen können auch im Zusammenhang mit verschiedenen Zeichenspielen die Funktion von Rollenübersetzungen haben. Dies ist schon beim Zusammenwirken der elementaren mathematischen Zeichenspiele zu beobachten. Beispielsweise kann das Zusammenwirken von Addition und Multiplikation durch die Rollenbeschreibung $\mathrm{a}+\mathrm{a}+\mathrm{a} \ldots+\mathrm{a}=p \cdot \mathrm{a}$ ( $p$ Summanden) oder das Zusammenwirken von Multiplikation und Potenzierung durch die Rollenbeschreibung, ,a $\cdot \mathrm{a}$. $\mathrm{a} \cdot \mathrm{a} \ldots \mathrm{a} \cdot \mathrm{a}^{\mathrm{a}}=\mathrm{a}^{\mathrm{n}}$ ( $n$ Faktoren) übersetzt werden.

Das Zeichenspiel „Algebra“ wird häufig zur Beschreibung von Rollen in anderen Zeichenspielen verwendet. Es kann etwa zur Beschreibung (Auswahl) von Rollen im Zeichenspiel Dezimalzahl (als Verkörperung der natürlichen Zahlen) verwendet werden. Beispiel: $a \in I N, a>7$. Rollenveränderungen, wie sie in Abschn. 4 im Zusammenhang mit dem Punkt erwähnt wurden, ergeben im Verlauf des Lernprozesses ständig neue Methoden der Beschreibung dieser Rollen. Beispiel: Hat man die Rolle einer geraden Linie als „Gerade“ etwa in der euklidischen Geometrie erlernt, so ändert sich die Rolle dieser geraden Linie etwa als lineare Funktion alleine dadurch, dass sie nun ein Ort unendlich vieler Punkte ist. Es gibt daher neue Möglichkeiten, mithilfe von Zeichenspielen wie Funktionsgleichung, Parameterform oder Normalvektorform diese neue Rolle der geraden Linie zu beschreiben. Häufig verwendet man das Zeichenspiel „Algebra“, auch um geometrische Inskriptionsverwendungen zu präzisieren. Beispiel: Die Herleitung der Tangentensteigung im Zusammenhang mit der Differentialrechnung beruht zunächst auf der geometrischen Andeutung der Tangente mithilfe von Sekanten. Verwendet man die Striche als Sekanten bzw. als Tangente, so ist an dieser Stelle die Rolle der Striche im Sinne der Steigung noch nicht präzisiert (Beispiel aus Brunner 2015b, S. 20) (Abb. 3).

Diese Rollenfestlegung wird durch entsprechende Rollenbeschreibungen letztlich algebraisch in Form des Differentialquotienten vorgenommen (Abb. 4). 
Abb. 3 So ist die Verwendung der Inskriptionen im Hinblick auf die Steigung noch nicht festgelegt

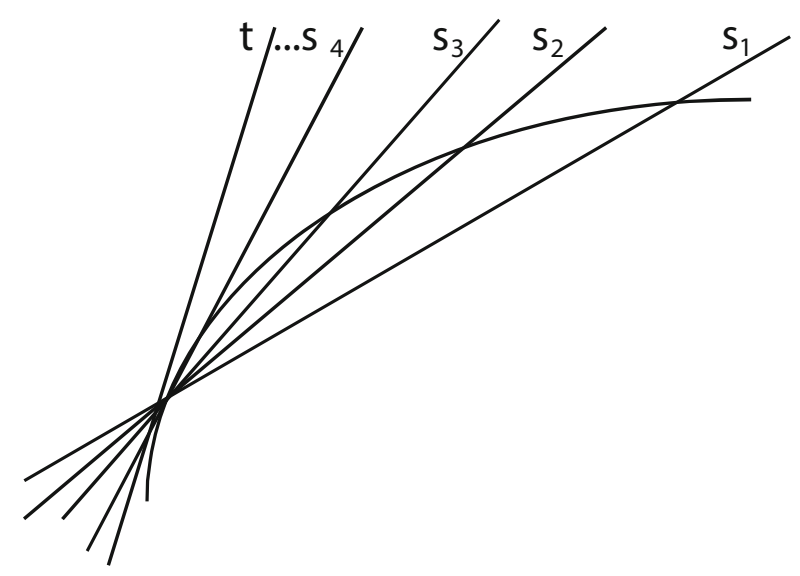

Abb. 4 Durch die Algebraisierung werden die Rollen der geraden Linien fixiert

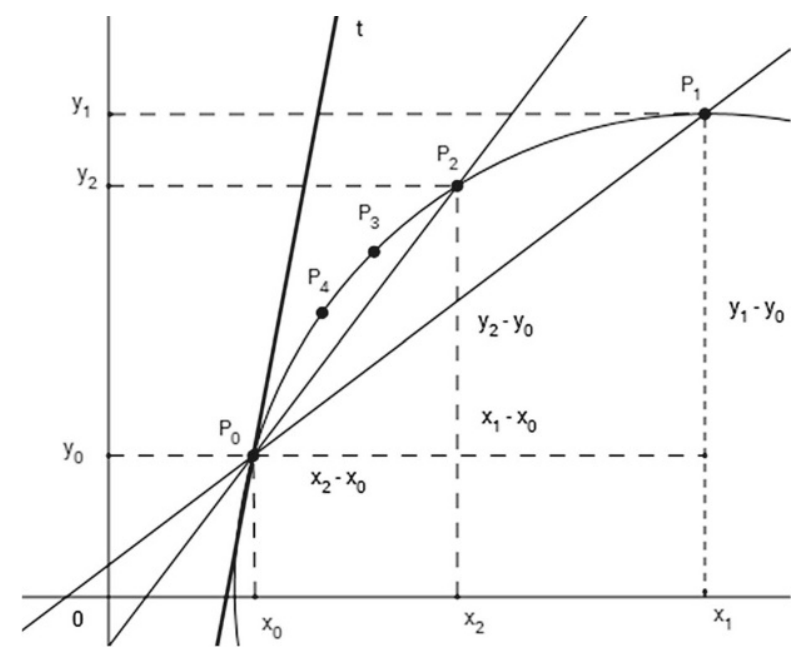

\section{Typ und Rollenübertragung}

Die Verwendung unterschiedlicher Inskriptionen nach gleichen Rollen ist eine Herausforderung mathematischen Tuns. Beispiel: $(a+b)^{2}=a^{2}+2 a b+b^{2} \sim(2 y+3 z)^{2}=$ $4 y^{2}+12 y z+9 z^{2}$. Man verwendet hier $2 y$ in der Rolle von a und $3 z$ in der Rolle von b. Diese Rollenübertragung ist eine Form der Verallgemeinerung. In Brunner (2013) wurde sie ausgehend vom ,token-type“-Konzept nach Peirce mithilfe der Inskriptionsverwendung erklärt. Nach Brunner (2013) handelt es sich bei solchen Typen um Äquivalenzklassen über Inskriptionen, die im Hinblick auf deren Verwendung gebildet werden. Die Bedeutung von Inskriptionen ist dabei genau im Hinblick auf einen bestimmten Typ die gleiche. Beispiele siehe Abb. 5 .

In der Diktion des vorliegenden Aufsatzes bildet man die Äquivalenzklassen im Hinblick auf bestimmte Rollen von Inskriptionen. Beim Beispiel (a) von Abb. 5 bedeutet dies etwa $2 \sim 3 \sim$ a und $3 \mathrm{i} \sim 7 \mathrm{i} \sim$ bi. Will man auf diese Art und Weise den gan- 
Abb. 5 Die angeführten Inskriptionen gehören im Hinblick auf die Verwendung als „komplexe Zahl“ (a), als „Dreieck“ (b) zur gleichen Äquivalenzklasse

\section{$2+3 i \sim 3+7 i \sim a+b i$}

a

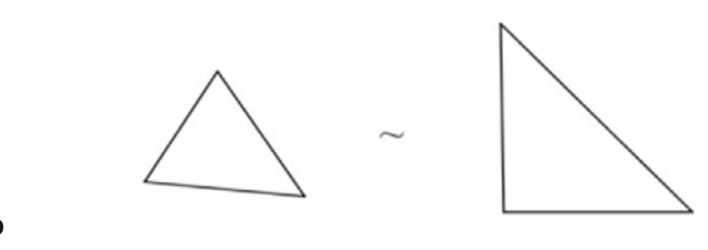

b

zen Zeichenspieltyp übertragen, so muss man in der Lage sein, die jeweiligen Rollen der Inskriptionen und die entsprechenden systemischen Regeln zu übertragen. Die Regeln der Rollen der Inskriptionen müssen dabei von den Lernenden selbst freigelegt werden. Die Didaktik der Vermittlung von Typen besteht im Wittgenstein'schen Sinn in der Erläuterung des Inskriptionsgebrauchs. Man führt entsprechende Beispiele an und beschreibt den Umgang mit den Inskriptionen. Nach den Prototypentheorien haben dabei erste Beispiele die Funktion von Prototypen. Beispiel: $\frac{2-z}{4-z^{2}}=\frac{1}{2+z} \sim$ $\frac{a-c}{a^{2}-c^{2}}=\frac{1}{a+c} \sim \frac{2 a-b}{4 a^{2}-b^{2}}=\frac{1}{2 a+b} \sim \frac{3 c-2 b}{9 c^{2}-4 b^{2}}=\frac{1}{3 c+2 b} \sim \frac{4+(-b-c)}{16-(b+c)^{2}}=\frac{1}{4+(b+c)}$ usw. Bei der Freilegung der äquivalenten Zeichenspiele müssen die jeweiligen Rollen der Inskriptionen und die systemischen Regeln durch den reflektierenden Vergleich mit den entsprechenden Prototypen decodiert werden. Welches Zeichenspiel als Prototyp genommen wird, ist prinzipiell egal. Möglicherweise wird man im obigen Fall ein ,einfacheres“" wie etwa $\frac{a-c}{a^{2}-c^{2}}=\frac{1}{a+c}$ nehmen.

Diese Sichtweise auf die angeführte Art der Bedeutungsherstellung ist didaktisch aufschlussreich. Hier ein Beispiel dazu: Die algebraische Inskription $(a+b)^{2}=a^{2}+$ $2 \mathrm{ab}+\mathrm{b}^{2}$ steht für zwei verschiedene Rollenbeschreibungen der Inskriptionen des in Abb. 6 angeführten geometrischen Zeichenspiels.

Die angeführte Sichtweise lässt etwa Zweifel im Zusammenhang mit dem Nutzen von geometrischen Veranschaulichungen algebraischer Formeln aufkommen. Geometrische Zeichenspiele wie das in Abb. 6 werden ja häufig als Verständnishilfen im Zusammenhang mit algebraischen Formeln wie hier $(a+b)^{2}=a^{2}+2 a b+b^{2}$ eingesetzt. Bei den angeführten algebraischen und geometrischen Darstellungen handelt es sich um vollkommen unterschiedliche Zeichenspiele. Es werden unterschiedliche Inskriptionen nach verschiedenen Rollen verwendet. Hier einige Details zu den Rollen- und Bedeutungsunterschieden: Im algebraischen Zeichenspiel stehen a und b für sich alleine betrachtet für nichts. Es ist nur wichtig, dass es verschiedene schreibregelkonforme Inskriptionen sind. Im Zusammenhang mit , $a+b$ “ ergibt die Formel nur dann Sinn, wenn bei den Ersetzungen von a und von b die Inskription „,+“ nicht verschwindet. Für $(2 \mathrm{a}+3 \mathrm{a})^{2}$ bräuchte man die Formel zum Beispiel nicht. Die Inskription „2“ steht als Hochzahl für die Ersetzungsregel $a^{2}=a \cdot a$. Sie dient der Zeichenökonomie. Von links nach rechts steht die Formel für die Klammerauflösung unter dem speziellen Umstand der hochgestellten „2“. Von rechts nach links steht die Formel für die Faktorisierung des angeführten Polynoms. Die Bedeutung der Formel resultiert weitgehend aus der Symmetrie, welche durch die hochgestell- 
Abb. 6 Die algebraischen Inskriptionen $(a+b)^{2}=a^{2}+2 a b+$ $b^{2}$ stehen für zwei verschiedene Möglichkeiten der Berechnung der Rechtecksflächen. Ist diese Darstellung eine Veranschaulichung der binomischen Formel?

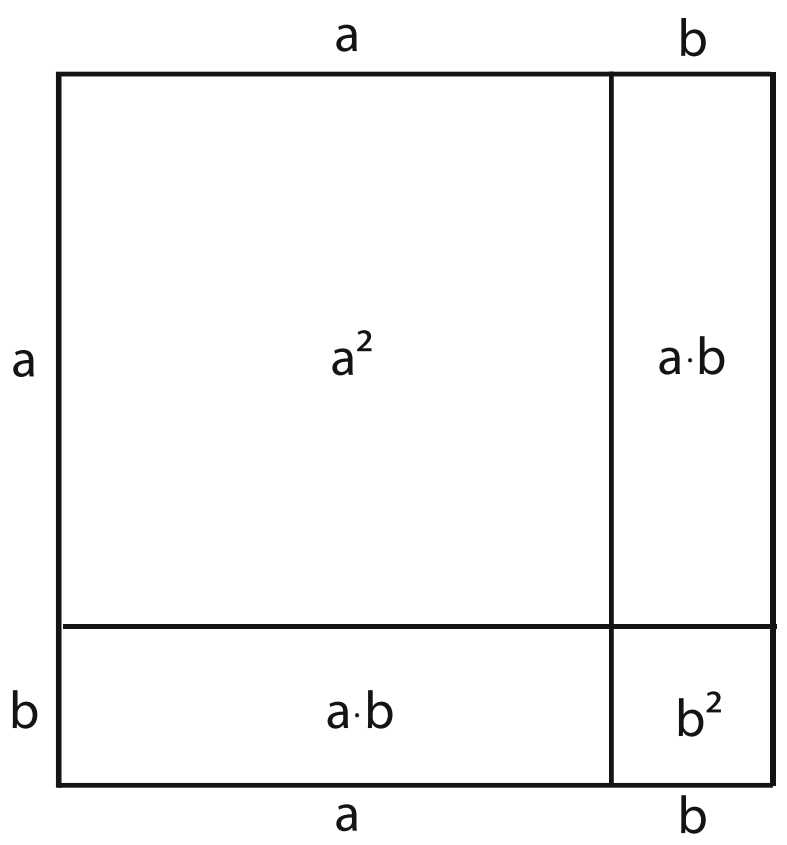

ten Zweien sichtbar wird, sowie aus dem operativen Vorteil, der sich gegenüber der Multiplikation $(a+b)(a+b)$ ergibt. Bei dem geometrischen Zeichenspiel haben a, $\mathrm{b}$ und $(\mathrm{a}+\mathrm{b})$ einmal die Rolle von Namen. Die verschiedenen geraden Linien sind dadurch im algebraischen Zeichenspiel unterscheidbar. Die Rolle der Linien ist jene von Anzahlen von Spalten bzw. Zeilen von Einheitsquadraten. Beim Zeichenspiel der Fläche steht $(a+b)^{2}=a^{2}+2 a b+b^{2}$ für zwei verschiedene Möglichkeiten der Zusammensetzung der Gesamtfläche durch Teilflächen. Es geht also um verschiedene Äquivalenzklassen und daher um unterschiedliche Typen. Es gilt einerseits: $(\mathrm{a}+$ b) $)^{2}=a^{2}+2 a b+b^{2} \sim(3 b+5 z)^{2}=9 b^{2}+30 b z+25 z^{2}$ usw. und andererseits siehe Abb. 7.

Die beiden in Abb. 8 angeführten Zeichenspiele gehören aber nicht zur selben Äquivalenzklasse.

Die angeführten Details zeigen deutlich, dass Verwendungsprobleme der Formel innerhalb der Algebra kaum durch geometrische Darstellungen wie in Abb. 7 und 8 erleichtert werden können. Diese müssen inneralgebraisch bearbeitet werden.

Nicht immer wird das ganze Zeichenspiel übertragen. Man muss auch in der Lage sein, nur einzelne Rollen von Inskriptionen zu übertragen. Bei speziellen Dreiecken wie dem rechtwinkligen, gleichschenkligen oder gleichseitigen Dreieck werden beispielsweise die Inskriptionen einerseits wie beim allgemeinen Dreieck verwendet. Es gilt zum Beispiel bei allen speziellen Dreiecken die bereits erwähnte Regel, dass das Zusammentreffen zweier Seiten als Eckpunkt verwendet wird. Andererseits gelten bei den Rollen von Inskriptionen auch spezielle Regeln. Beim gleichseitigen Dreieck eben jene, dass alle Seiten als gleich lang verwendet werden. Man muss also im Zusammenhang mit einem speziellen Dreieckstyp 
Abb. 7 Die beiden Darstellungen sind vom gleichen Typ
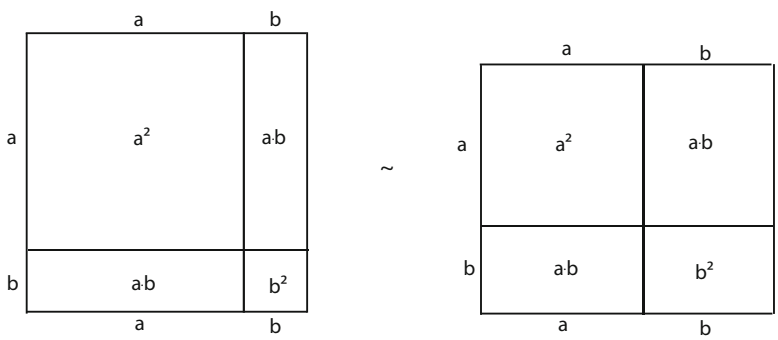

Abb. 8 Die beiden Darstellungen sind nicht vom gleichen Typ

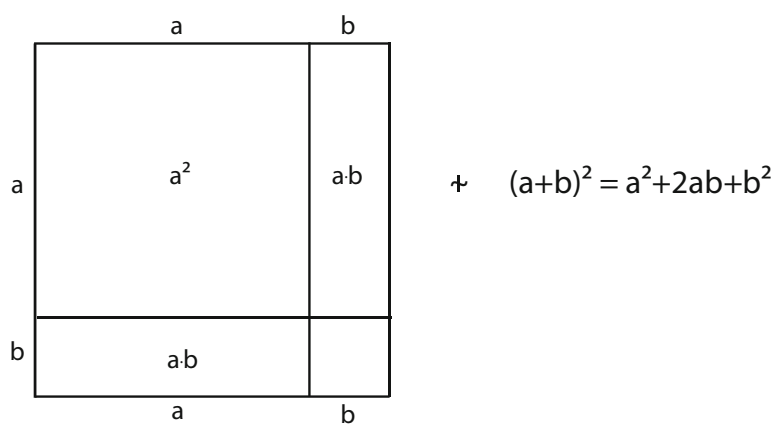

in der Lage sein, spezielle Rollen von Inskriptionen von den Rollen im allgemeinen Dreieck zu separieren. Bei der Übertragung von Rollen muss im Sinne der meist erforderlichen multiplen Rollenverwendung ständig auf die Verwendung der Inskriptionen in anderen Rollen geachtet werden. Es werden ja häufig verschiedene Zeichenspiele miteinander kombiniert. Beim nachfolgend angeführten Ausschnitt aus der Herleitung der Produktregel der Differentialrechnung werden etwa die Zeichenspiele , $\mathrm{a}=\mathrm{a}+\mathrm{c}-\mathrm{c}^{*}$ und , $f(\mathrm{x})=\mathrm{u}(\mathrm{x}) \mathrm{v}(\mathrm{x})^{\text {" }}$ miteinander kombiniert: $\frac{f(z)-f(x)}{z-x}=\frac{u(z) v(z)-u(x) v(z)+u(x) v(z)-u(x) v(x)}{z-x}=\frac{u(z)-u(x)}{z-x} \cdot v(z)+u(x) \cdot \frac{v(z)-v(x)}{z-x}$.

\section{Epistemologisches Potential des Rollenwechsels}

Die epistemologische Bedeutung der Verwendung unterschiedlicher Inskriptionen in gleichen Rollen wurde bereits im vorigen Abschnitt im Zusammenhang mit dem Konzept des Typs angedeutet. Es können aber auch gleiche Inskriptionen in unterschiedlichen Rollen verwendet werden (vgl. etwa Abschn. 3). Auch diese Verwendungsweise bietet vielfältige epistemologische Möglichkeiten. Hier nun einige Beispiele dafür. Generell belegen die verschiedenen im vorliegenden Artikel angeführten Beispiele, dass mithilfe der Sichtweisen Rolle und Zeichenspiel das epistemologische Potential mathematischer Darstellungen gut beleuchtet werden kann.

Im Zusammenhang mit der Ermittlung von Lösungen für gestellte Probleme geht es in der Mathematik zunächst einmal um die Wahl geeigneter Zeichenspiele. Im Verlauf der Problemlösung kann es sich als zweckmäßig herausstellen, die Sichtweise auf gewählte Zeichenspiele zu ändern. Der geschickte Wechsel der Sichtweise hat epistemologisches Potential. Für Hoffmann (2005, S. 177) ist der ,Wechsel des 
Abb. 9 Der Satz von Desargues anschaulich dargestellt: Zwei Ebenen schneiden sich in einer Geraden. (Grafik ähnlich wie bei Hilbert und Cohn-Vossen (1932) 1996; erstellt mit GeoGebra)

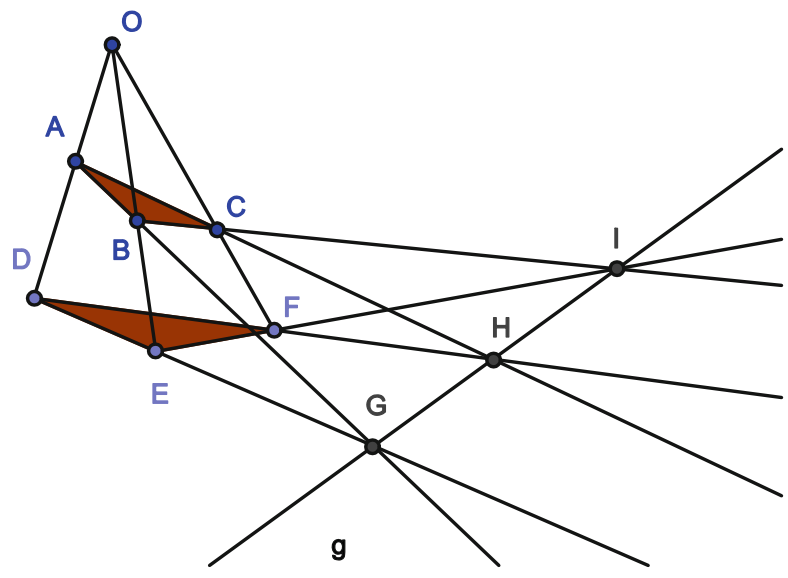

Blickpunktes“ das entscheidende Merkmal für den Begriff der ,theorischen Transformation“. Als Beispiel behandelt Hoffmann, so wie schon Peirce, den berühmten Beweis des Satzes von Desargues über zwei in einer Abbildung liegende Dreiecke (siehe Abb. 9). Die von Hoffmann angeführte Beweismethode von Staud beruht auf der Änderung der Sichtweise. Die beiden Dreiecke werden nicht in der Ebene, sondern in räumlicher Perspektive, also dreidimensional, wahrgenommen. Die entscheidende Idee, nämlich die Einführung eines außerhalb der Ebene angenommenen Perspektivpunktes ist nach Hoffmann ein kreativer Akt „theorematischer Deduktion“. Der Rest ist nach Peirce korollares Schließen ,mittels einiger weniger offensichtlicher, in der Theorie der Perspektive bekannter Definitionen“ (zitiert bei Hoffmann 2005, S. 176).

In der Diktion des vorliegenden Aufsatzes ist die entscheidende Idee des angeführten Beweises der Wechsel der Rolle der Inskriptionen durch deren Verwendung in räumlicher Perspektive als ,Dreieck in der Ebene“, „Punkt in der Ebene“ oder „Gerade in der Ebene“. Die Einführung eines außerhalb der Ebene angenommenen Punktes in der Rolle „Projektionszentrum“ ändert das gesamte Zeichenspiel. Im Zusammenhang mit dieser Art des Sichtweisenwechsels sei auf eine bedeutsame Konvention der Mathematik verwiesen: Es erscheint bei dieser Art der Beweisführung bemerkenswert, dass ein räumlich geführter Beweis von der Community der Mathematiker/ innen als Beweis für einen Satz der Ebene akzeptiert wird. Es handelt sich doch um unterschiedliche Zeichenspiele, unterschiedliche Rollen und damit um unterschiedliche Bedeutungen gleicher Inskriptionen. Die Gleichheit der verwendeten Inskriptionen erscheint in der Mathematik also als Garant für Gleichheit. Dies scheint hierbei die Konvention zu sein. Hier noch ein algebraisches Beispiel zum gleiche Phänomen des Sichtweisenwechsels. Auch hier ist neben dem Wechsel des Zeichenspiels auch die Verwendung gleicher Inskriptionen nach anderen Rollen zu beobachten: $\int\left(\sin ^{2} x\right) \mathrm{d} x=\int(\sin x)(\sin x) \mathrm{d} x=(-\cos x) \sin x-\int(-\cos x)(\cos x) \mathrm{d} x=$ $(-\cos x) \sin x+\int(\cos x)^{2} \mathrm{~d} x=(-\cos x) \sin x+\int\left(1-\sin ^{2} x\right) \mathrm{d} x=(-\cos x) \sin x+$ $x-\int\left(\mathrm{n}^{2} x\right) \mathrm{d} x$. Also: $\int\left(\sin ^{2} x\right) \mathrm{d} x=\frac{-\cos x \cdot \sin x+x}{2}$. Bei diesem Beispiel steht etwa das zweite Gleichheitszeichen für einen Wechsel des Zeichenspiels (partielle Integration). Vor dem zweiten Gleichheitszeichen haben die Inskriptionen „sinx“ 
Abb. 10 Die kreative Veränderung der Linien (gestrichelte Linien) führt im Zeichenspiel „Umfang“ zu einer Erkenntnis: $\mathrm{u}=2 \mathrm{x}+2 \mathrm{z}$

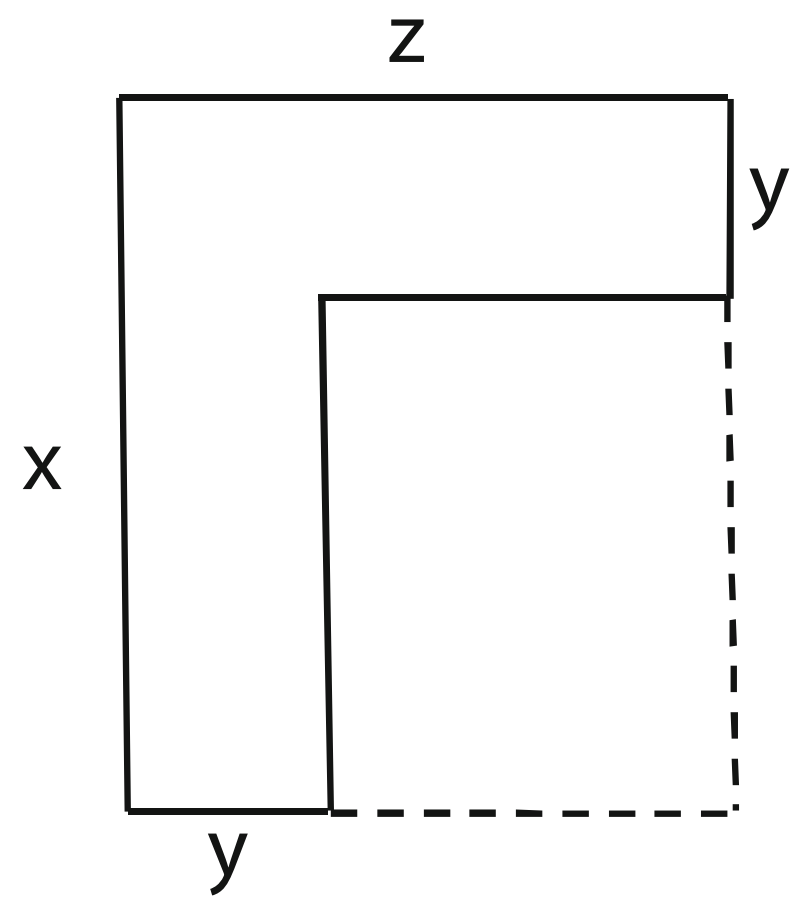

noch die gleiche Bedeutung. Nach dem zweiten Gleichheitszeichen sind sie durch verschiedene Inskriptionen nach Rollen des „,neuen“ Zeichenspiels ersetzt. Weitere Ersetzungen von Inskriptionen beruhen auf Rollenbeschreibungen wie: $\sin ^{2} x=$ $(\sin x)(\sin x) ; \cos ^{2} x=(\cos x)(\cos x) ; \cos ^{2} x=1-\sin ^{2} x$. Auch Schreibregeln wie $\sin ^{2} x=(\sin x)^{2}$ kommen vor.

Das kreative Verändern/Verschieben/Austauschen von Inskriptionen im Hinblick auf bestimmte Rollen von Inskriptionen hat ebenfalls großes epistemologisches Potential. Die Wahl der Zeichenspiele wird dabei durch die zu erreichenden Ziele bestimmt. Beim folgenden Beispiel aus Brunner (2015b, S. 18) ist im Hinblick auf das Zeichenspiel „Umfang“ die Veränderung der entsprechenden Linien zu den jeweils gestrichelten Linien eine erkenntnisreiche. Mithilfe dieses einfachen geometrischen Experiments kann man leicht erkennen, dass der Umfang von y unabhängig ist. Es entstehen quasi neue Linien, die wiederum nach gleichen Rollen verwendet werden: $(y+$ gestrichelte Linie 1$)=x$ und $(y+$ gestrichelte Linie 2$)=z$. Es gilt damit: , $\mathrm{u}=2 \mathrm{x}+2 \mathrm{z}^{\prime \prime}$ ( siehe Abb. 10). Die Rolle der Linien ist jene der Anzahl von Einheitslängen. Umgekehrt kann die algebraische Transformation, ,z + y + (z-y) + $(x-y)+y+x=2 z+2 x$ “ als Hinweis darauf gesehen werden, dass diese Kenntnis auch geometrisch herstellbar sein muss. Diese angeführten Verschiebungen wären etwa im Hinblick auf das Zeichenspiel „Fläche“ sinnlos.

Hier noch ein anderes bekanntes Beispiel zur geschickten Manipulation mit Inskriptionen und deren Rollen. Es wird dem jungen Gauß zugeschrieben: Versucht man etwa die natürlichen Zahlen von 1 bis 100 zusammenzuzählen, so wird durch 
die geschickte Manipulation mit den Inskriptionen $1+2+3+\ldots+99+100=$ $(1+99)+(2+98)+\ldots+(49+51)+50=50 \cdot 100+50$ der Übergang von Additionen zu Multiplikationen (Rollenwechsel) ermöglicht. Der operative Vorteil von ,, $50 \cdot 100+50 “$ ist gegenüber ,,(( $1+2)+3)+4)+\ldots$ “. unübersehbar.

\section{Rollennachweise und Rollenhinweise}

Für die Erklärung der Funktionsweise mathematischer Definitionen, Sätze oder Beweise kann die vorgeschlagene Sichtweise von Zeichenspiel und Rolle ebenfalls von großem Nutzen sein. Hier zwei Beispiele:

Viele mathematische Sätze basieren auf so genannten Existenzbeweisen. Bei solchen Beweisen wird etwa nachgewiesen, dass unter bestimmten Voraussetzungen eine Nullstelle (Zwischenwertsatz), eine weitere Primzahl (Primzahlensatz von Euklid), eine Stelle z mit $f^{\prime}(z)=0$ (Satz von Rolle) usw. existiert. Derartige Sätze können als Rollennachweise betrachtet werden. Im Gegensatz zur etablierten Sichtweise, nach welcher bei derartigen Beweisen die Existenz abstrakter Objekte wie Stellen, Nullstellen, Primzahlen usw. unter bestimmten Voraussetzungen nachgewiesen wird, wird nach der unterlegten Sichtweise des vorliegenden Aufsatzes lediglich die Verwendbarkeit von Inskriptionen in bestimmten Rollen unter bestimmten Voraussetzungen nachgewiesen. Betrachten wir als Beispiel den Primzahlensatz nach Euklid. Es wird in diesem Satz folgender Nachweis gebracht: „Es gibt mehr Primzahlen als jede vorgelegte Anzahl von Primzahlen“. Der entsprechende Beweis wird mittels Widerspruch geführt. Es wird zunächst angenommen, dass es in einem zur Verkörperung der natürlichen Zahlen geeigneten Zeichensystem (etwa 0, 1, 2, 3, ...) nur endlich viele Inskriptionen $\mathrm{p}_{1}, \mathrm{p}_{2}, \ldots, \mathrm{p}_{\mathrm{n}}$ in der Rolle „Primzahl“ gibt. In der Folge wird angenommen, dass $m$ die kleinste Zahl, die von allen diesen Zahlen geteilt wird, also das Produkt aller dieser Zahlen (oder ein Vielfaches davon) sei. Betrachtet man dann den Nachfolger von $m$, nämlich $m+1$, so gibt es zwei Möglichkeiten: a) $m+1$ erfüllt selbst die Regeln der Rolle „Primzahl“. Aufgrund der Konstruktion ist $\mathrm{m}+1$ ist dann größer als $\mathrm{p}_{1}, \mathrm{p}_{2}, \ldots, \mathrm{p}_{\mathrm{n}}$. Dies steht im Widerspruch zur Annahme. b) $m+1$ erfüllt die Regeln der Rolle „Primzahl“ nicht. Auch diese Annahme führt bekanntlich zu einem Widerspruch (hier nicht ausgeführt). Es ist also nachgewiesen, dass es zu jeder vorgegeben Anzahl von Inskriptionen eines Zeichensystems wie etwa $0,1,2,3$,..., welche die Regeln des Zeichenspiels ,natürliche Zahlen“ und der Rolle „Primzahl“ erfüllen, weitere Inskriptionen in der Rolle „Primzahl“ geben muss.

Trotz des auftretenden Wortspiels ist der Satz von Rolle im obigen Sinne ein „Rollennachweis“: Aufgrund von bestimmten Bedingungen wie ,eine stetige reelle Funktion nimmt auf einem abgeschlossenen Intervall $[\mathrm{a}, \mathrm{b}]$ jeden Wert zwischen $f(\mathrm{a})$ und $f(\mathrm{~b})$ an" und , $f(\mathrm{a})$ und $f(\mathrm{~b})$ haben verschiedene Vorzeichen" wird die Existenz von mindestens einer Nullstelle von $f$ in $[\mathrm{a}, \mathrm{b}]$ garantiert, $\mathrm{d}$. h. von mindestens einer reellen Zahl werden die Regeln der Rolle „Nullstelle“ erfüllt.

Die vorgeschlagenen Sichtweisen Rolle und Zeichenspiel können auch als Erklärungshilfe für spezielle Beziehungen mathematischer Sätze zu den jeweils subsumierbaren Spezialfällen dienen. Beispiel: Nach Dörfler (2014, S. 40) treten die 
konkreten Darstellungen in vielen Sätzen über Funktionen selbst nicht auf. Es wird nur auf sie hingewiesen. Dörfler (2014, S. 40) schreibt:

Für diese Definitionen und die daran anschließenden Sätze und Beweise sind die verschiedenen Darstellungen von Funktionen (Graph, Formel, Tabelle, Funktiontionalgleichung) gänzlich ohne Bedeutung [...] Soll jedoch die Stetigkeit einer speziellen Funktion nachgewiesen werden, so braucht man irgendeine Darstellung, etwa die Reihendarstellung der Exponentialfunktion.

Zeichenspiele wie Funktionen können in mathematischen Texten unterschiedlich genau fixiert sein. Im Falle von Formulierungen wie , $f$ sei eine Funktion“ sind die Rollen der involvierten Inskriptionen nur sehr allgemein festgelegt. Es gelten Regeln wie etwa: Zu jedem x des Definitionsbereichs von $f$ gibt es genau ein $f(\mathrm{x})$. Bei einer Formulierung wie ,,sei $f$ die Funktion mit $\mathrm{x} \rightarrow \mathrm{ax}^{2}+\mathrm{b}^{\text {“ }}$ ist der Grad der Festlegung größer. Es ist der Typ des Zeichenspiels angegeben. Schreibt man aber ,sei $f$ die Funktion mit $x \rightarrow 3 x^{2}-1$ “, so ist die Rollenfestlegung für „Wissende“ sehr genau. Darstellungen wie Funktionsgleichung oder Funktionsgraph können genau ermittelt werden. Vom allgemeinen zum speziellen Fall nimmt die Anzahl der geltenden Regeln jeweils zu. Die Möglichkeit, Zeichenspiele sehr allgemein anzugeben, hilft bei der Formulierung von Definitionen, Sätzen und Beweisen. Es gelten nur verhältnismäßig wenige Verwendungsregeln. Die jeweiligen Rollen sind nur sehr allgemein fixiert. Viele Spezialfälle sind subsumierbar. Soll aber im Sinne des obigen Zitats ein Rollennachweis wie etwa die Stetigkeit für eine spezielle Funktion nachgewiesen werden, so muss das Zeichenspiel der Definition erst an die Rollen der speziellen Inskriptionen angepasst werden. Diese Übersetzungsleistung ist eine anspruchsvolle. Es gelten spezielle Verwendungsregeln. Hier ein konkretes Beispiel: Es soll die Stetigkeit der Funktion $f(x)=3 x^{2}-1$ an der Stelle $\mathrm{z} \in \mathrm{D}$ gezeigt werden. Die Rolle „stetig“ wird im angeführten Kontext durch das nachfolge Zeichenspiel „EpsilonDelta-Definition“ festgelegt: Die Funktion $f: \mathrm{D} \rightarrow \mathrm{IR}$ ist stetig in $\mathrm{z} \in \mathrm{D}$, wenn (Zeichenspiel:) zu jedem $\varepsilon>0$ ein $\delta>0$ existiert, sodass für alle $\mathrm{x} \in \mathrm{D}$ mit $|\mathrm{x}-\mathrm{z}|<$ $\delta$ gilt: $|f(\mathrm{x})-f(\mathrm{z})|<\varepsilon$. Die Erfüllung der beschriebenen Rolle ,stetig“ ist für alle subsumierbaren Inskriptionen des vorausgesetzten Zeichenspiels „Funktion“ separat $\mathrm{zu}$ belegen. Will man nachweisen, dass $f(\mathrm{x})=3 \mathrm{x}^{2}-1$ in $\mathrm{z} \in \mathrm{D}$ stetig ist, so ist die Deutung der Rolle der Inskriptionen und deren Zusammenwirken im gegebenen Zeichenspiel eine Herausforderung. Neben einfachen Ersetzungen wie $|f(\mathrm{x})-f(\mathrm{z})|=$ $\left|3 x^{2}-1-\left(3 z^{2}-1\right)\right|=\left|3 x^{2}-3 z^{2}\right|$ ist vor allem die Übersetzung des Zusammenwirkens von $|\mathrm{x}-\mathrm{z}|<\delta$ mit $|f(\mathrm{x})-f(\mathrm{z})|<\varepsilon$ eine anspruchsvolle. Die Abhängigkeit der Rollen von $\varepsilon$ und $\delta$ nach den geltenden Regeln muss mithilfe von geschickten algebraischen Transformationen nachgewiesen werden: Nimmt man an, dass $|x-z|<1$ ist, dann ist $|\mathrm{x}+\mathrm{z}|=|2 \mathrm{z}+(\mathrm{x}-\mathrm{z})| \leq 2|\mathrm{z}|+1$. Damit erhält man $|f(\mathrm{x})-f(\mathrm{z})|=\mid 3 \mathrm{x}^{2}-1-$ $\left(3 z^{2}-1\right)|=| 3 x^{2}-3 z^{2}|=3| x+z|\cdot| x-z|\leq 3(2|z|+1) \cdot| x-z \mid<\varepsilon$ für alle $x$ mit $|x-z|<\varepsilon / 3(2|z|+1)$. Für $\varepsilon>0$ gilt $\delta(\varepsilon)=\operatorname{Min}(1, \varepsilon / 3(2|z|+1))$. Für die jeweiligen Rollennachweise ist ein hohes Maß an Vertrautheit mit dem Zeichenspiel Algebra erforderlich. 


\section{Rolle und Erfahrungswelt}

Beim Einsatz der „Rolle“ als Erklärungsweise kann die Erfahrungswelt der Lernenden einbezogen werden. Lernen durch Analogiebildung ist nach Metzig und Schuster (2000) ein fast müheloses, da neues Wissen auf der Basis von altem generiert wird. Dieses Lernen garantiert ebenfalls nach Metzig und Schuster (2000) langfristiges Behalten, verbessertes Verständnis für den Wissensbereich, mehr Kreativität, da das alte Wissen neue Hypothesen über den neuen Wissensbereich erlaubt, emotionale Ankopplung usw. Es hängt natürlich vom Geschick der Lehrenden ab, durch geeignete Analogien derartige positive Effekte zu erwirken und nicht durch ungeeignete Vergleiche Missverständnisse zu erzeugen. Im Folgenden sind nun Vorschläge für sinnvolle Analogien zwischen den Rollen der Mathematik und den Rollen der Erfahrungswelt der Lernenden angeführt.

Lernende und Lehrende sind aus der eigenen Erfahrungswelt mit „Rollen“ in unterschiedlichen Kontexten vielfältig vertraut. Sie sind es gewohnt, in den verschiedenen „Zeichenspielen des Alltags" Rollen wie etwa jene als Schulsprecherin, Tochter, Mutter, Tante, Lehrerin, Pfadfinderin usw. zu spielen. Sie sind auch an den Gebrauch von Wörtern oder Gegenständen in verschiedenen Rollen gewöhnt. Sie sind es sogar gewohnt, Gegenstände, deren Rollen genau fixiert zu sein scheinen, in fremden Rollen zu verwenden. Beispielsweise gibt es „Notfälle“, in welchen sie etwa eine Zange als „Hammer“ oder ein Stück Karton als „Regenschirm“ verwenden. Sie sind es möglicherweise auch gewohnt, gleiche Inskriptionen innerhalb und außerhalb der Mathematik in verschiedenen Rollen zu verwenden. Eine Inskription wie ,6/8“ wird etwa im Zeichenspiel der Notenschrift in einer anderen Rolle als als Bruch gebraucht. Bei fast allen Tätigkeiten des täglichen Lebens müssen Lernende und Lehrende also in der Lage sein, Rollen im Kontext mit verschiedensten Zeichenspielen zu beachten. Es gibt also eine breite Palette von möglichen Analogien, die im Mathematikunterricht gewinnbringend eingesetzt werden können. Im Folgenden sind nun einige Beispiele für derartige Analogien angeführt.

Eine Möglichkeit der Analogiebildung besteht durch die Regelgebundenheit der Rolle im Vergleich von Regeln. Rollenbesonderheiten können so veranschaulicht werden. Es kann beispielsweise der Unterschied zwischen Grundregeln und abgeleiteten Regeln beleuchtet werden. Auf die Unterscheidung von Grundregeln und abgeleiteten Regeln wurde im Verlauf des Aufsatzes im Zusammenhang mit mathematischen Inskriptionsverwendungen bereits hingewiesen. Regelfolgen ist übrigens auch ohne Verständnis der Regelableitung möglich. Beispielsweise kann man bei entsprechender Vertrautheit den pythagoreischen Lehrsatz auch dann erfolgreich benutzen, wenn man ihn nicht beweisen kann. Zusammenhänge wie diese sind auch im Alltag beobachtbar. Die Regel, dass Verkehrsteilnehmer auf der rechten Seite der Straße fahren müssen, ist beispielsweise eine Grundregel. Aus der Gültigkeit dieser Regel resultieren andere Regeln. Beispielsweise jene, dass man links überholen muss. Diese abgeleitete Regel kann auch ohne Verständnis für den Sinn ihrer Einführung befolgt werden.

Es ist nicht nur möglich Regelbesonderheiten mithilfe von Analogien zu erläutern, man kann so gut wie alle Formen der Bedeutungsfestlegung und Bedeutungsveränderung, die in den vergangenen Abschnitten des vorliegenden Aufsatzes im 
Zusammenhang mit der Rolle von Inskriptionen thematisiert wurden, in Analogie mit Beispielen aus dem Alltag beleuchten. Hier zwei Beispiele: Als Analogie zum Rollenwechsel in der Mathematik (Abschn. 3, 5 und 8) könnte man etwa Spielkarten, Schachfiguren oder Verkehrszeichen verwenden. Dieselben Spielkarten werden etwa in den Kartenspielen Rommé, Kanaster oder Bridge nach unterschiedlichen Rollen verwendet. Beim Schachspiel könnte man „Dame“ und „König“ oder bei verschiedenen Kartenspielen „Unter“ und „Ober“ ohne Probleme vertauschen. Wenn die Spielenden damit einverstanden wären, bereitete dies keine Probleme. Analog könnte das Verkehrszeichen „Vorrang geben“ etwa mit jenem für „Stopp“ getauscht werden. Als Analogie zum Rollennachweis in der Mathematik könnte man als skurrile Analogie einen „Mordnachweis“ wie den folgenden bringen: In einem Krimi wird eine Leiche gefunden. Zunächst wird ein Unfall vermutet. Aufgrund von Untersuchungen stellt man aber fest, dass es Mord sein muss. Es treten also Bedingungen ein, welche die Verwendung des Wortes „Mörder/in“ rechtfertigen. Es muss mindestens eine Person in dieser Rolle geben. Nach dieser Person kann/muss nun auch gesucht werden. Dem Einfallsreichtum der Lehrenden sind im Zusammenhang mit erklärenden Analogien aus dem Alltag keine Grenzen gesetzt.

\section{Fazit}

Die Rolle von Inskriptionen in mathematischen Zeichenspielen kann als nützliche Sichtweise sowie als allgemeines Beschreibungs- und Erklärungsmodell im Zusammenhang mit der Funktionsweise und dem Zusammenwirken von mathematischen Darstellungen und dem Sprechen über mathematische Darstellungen im Schulunterricht eingesetzt werden. Es ergeben sich viele Analogien, welche zur Verständnisförderung im Zusammenhang mit mathematischen Phänomenen wie etwa der Möglichkeit der epistemologischen Nutzung mathematischer Darstellungen verwendet werden können. Die angeführte Sichtweise erlaubt eine allgemeine Außensicht, mit deren Hilfe verschiedenste Bezüge zur Erfahrungswelt der Lernenden hergestellt werden können.

Open access funding provided by Paris Lodron University of Salzburg.

Open Access Dieser Artikel wird unter der Creative Commons Namensnennung 4.0 International Lizenz (http://creativecommons.org/licenses/by/4.0/deed.de) veröffentlicht, welche die Nutzung, Vervielfältigung, Bearbeitung, Verbreitung und Wiedergabe in jeglichem Medium und Format erlaubt, sofern Sie den/die ursprünglichen Autor(en) und die Quelle ordnungsgemäß nennen, einen Link zur Creative Commons Lizenz beifügen und angeben, ob Änderungen vorgenommen wurden.

\section{Literatur}

Bedürftig, Th. (2016). Was ist ein Punkt? - Ein Streifzug durch die Geschichte. In R. Krömer \& G. Nickel (Hrsg.), Siegener Beiträge zur Geschichte und Philosophie der Mathematik (S. 1-21).

Brunner, M. (2009). Lernen von Mathematik als Erwerb von Erfahrungen im Umgang mit Zeichen und Diagrammen. Journal für Mathematik-Didaktik, 30(3/4), 206-231.

Brunner, M. (2011). Ständige Restrukturierung - ein Erfordernis des Lernens von Mathematik. mathematica didactica, 34, 20-49. 
Brunner, M. (2013). Didaktikrelevante Aspekte im Umfeld der Konzepte token und type. Journal für Mathematik-Didaktik, 34(1), 53-72.

Brunner, M. (2015a). Diagrammatische Realität und Regelgebrauch. In G. Kadunz (Hrsg.), Semiotische Perspektiven auf das Lernen von Mathematik (S. 33-49). Berlin Heidelberg: Springer.

Brunner, M. (2015b). Bedeutungsherstellung als Lehr- und Lerninhalt. mathematica didactica, 38, 199-223.

Dawydow, W. (1977). Arten der Verallgemeinerung im Unterricht. Berlin: Volk und Wissen.

Dörfler, W. (1988). Begriff als Tätigkeitsstruktur - Zur Unterscheidung von empirischem und theoretischem Begriff. In P. Brender (Hrsg.), Mathematikdidaktik: Theorie und Praxis (S. 29-36). Berlin: Cornelsen.

Dörfler, W. (2006). Diagramme und Mathematikunterricht. Journal für Mathematik-Didaktik, 27(3/4), 200-219.

Dörfler, W. (2013). Was würden Peirce oder Wittgenstein zu Kompetenzmodellen sagen? In M. Rathgeb, M. Helmerich, R. Krömer, K. Lengnink \& G. Nickel (Hrsg.), Mathematik im Prozess. Philosophische, Historische und Didaktische Perspektiven (S. 73-87). Wiesbaden: Springer Spektrum.

Dörfler, W. (2014). Didaktische Konsequenzen aus Wittgensteins Philosophie der Mathematik. In H. Hahn (Hrsg.), Anregungen für den Mathematikunterricht unter der Perspektive von Tradition, Moderne und Lehrerprofessionalität (S. 68-80). Berlin: Franzbecker.

Dörfler, W. (2015). Abstrakte Objekte in der Mathematik. In G. Kadunz (Hrsg.), Semiotische Perspektiven auf das Lernen von Mathematik (S. 33-49). Berlin Heidelberg: Springer.

Dörfler, W., \& Kadunz, G. (2006). Rezension von „Erkenntnisentwicklung“. Journal für MathematikDidaktik, 27(3/4), 300-318.

Epple, M. (1994). Das bunte Geflecht der mathematischen Spiele. Mathematische Semesterberichte, 41, $113-133$.

Hilbert, D., \& Cohn-Vossen, St. (1996). Anschauliche Geometrie. Berlin Heidelberg: Springer.

Hoffmann, A. (2007). Wittgensteins Regelbegriff. http://www.cse.unsw.edu.au/ achim/Research/ Philosophie/node68.html. Zugegriffen: 12. Okt 2012.

Hoffmann, M. (2005). Erkenntnisentwicklung. Philosophische Abhandlungen, Bd. 90. Frankfurt: Klostermann.

Krämer, S. (2009). Operative Bildlichkeit. Von der „Grammatologie“ zu einer „Diagrammatologie“? Reflexionen über erkennendes „Sehen“. In M. Hessler \& D. Mersch (Hrsg.), Logik des Bildlichen. Zur Kritik der ikonischen Vernunft (S. 94-121). Bielefeld: transcript.

Lakoff, G. (1987). Women, fire and dangerous things. What categories reveal about the mind. Chicago, London: The University of Chicago Press.

Mahr, B., \& Robering, K. (2009). Diagramme als Bilder, die Modelle repräsentieren: Diagrammgebrauch in der Elementargeometrie. Zeitschrift für Semiotik, 31(3/4), 275-310.

Metzig, W., \& Schuster, M. (2000). Lernen zu lernen. Heidelberg, Berlin: Springer.

Meyer, M. (2010). Wörter und ihr Gebrauch - Analyse von Begriffsbildungsprozessen im Mathematikunterricht. In G. Kadunz (Hrsg.), Sprache und Zeichen (S. 49-80). Hildesheim, Berlin: Franzbecker.

Mühlhölzer, F. (1999). Mathematik ohne Metaphysik. In J. Nida-Rümelin (Hrsg.), Rationalität, Revision. Vorträge des 3. Internationalen Kongresses der Gesellschaft für Analytische Philosophie, München, 15.-18. Sept 1997 (S. 416-423). Berlin: de Gruyter.

Mühlhölzer, F. (2012). On live and dead signs in mathematics. In M. Detlefsen \& G. Link (Hrsg.), Formalism and beyond. On the nature of mathematical discourses. Frankfurt: Ontos.

Nagl, L. (1992). Charles Sanders Peirce. Frankfurt New York: Campus.

Peschek, W. (1989). Abstraktion und Verallgemeinerung im mathematischen Lernprozess. Journal für Mathematikdidaktik, 10(3), 211-285.

Rosch, E. (1975). Cognitive reference points. Cognitive Psychology, 7, 532-547.

Rotman, B. (2000). Mathematics as Sign. Writing, Imaging, Couting. Stanford: University Press.

Schweiger, F. (2015). Klammern: Notwendig und unauffällig. In G. Kadunz (Hrsg.), Semiotische Perspektiven auf das Lernen von Mathematik (S. 135-139). Berlin, Heidelberg: Springer.

Stjernfelt, F. (2007). Diagrammatology - an investigation on the borderlines of phenomenology, ontology, and semiotics. Dordrecht: Springer.

Wittgenstein, L. (1978). Wittgensteins Vorlesungen über die Grundlagen der Mathematik, Bd. 7. Frankfurt am Main: Suhrkamp.

Wittgenstein, L. (1984a). Philosophische Grammatik. Werksausgabe, Bd. 4. Frankfurt am Main: Suhrkamp.

Wittgenstein, L. (1984b). Bemerkungen über die Grundlagen der Mathematik (2013. Aufl.). Werkausgabe, Bd. 6. Frankfurt am Main: Suhrkamp.

Wittgenstein, L. (2003). Philosophische Untersuchungen. Frankfurt: Suhrkamp. 